Review

\title{
Regulation of Membrane Phospholipid Homeostasis in Neurodegenerative Diseases
}

XinRan Quan, Marica Bakovic *

University of Guelph, Department of Human Health and Nutritional Sciences, Guelph Canada; EMails: xrquan.cq@gmail.com; mbakovic@uoguelph.ca

* Correspondence: Marica Bakovic; E-Mail: mbakovic@uoguelph.ca

Academic Editor: Michael Fossel

Special Issue: $\underline{\text { Research on Neurodegenerative Diseases }}$

OBM Geriatrics

2021, volume 5 , issue 3

doi:10.21926/obm.geriatr.2103176
Received: December 21, 2020

Accepted: July 13, 2021

Published: July 21, 2021

\begin{abstract}
Absrtact
Neurodegenerative diseases (NDs) are a diverse group of neuropathological diseases that are currently incurable due to the irreversible neuronal loss. At the present rate of the world population growth, it is projected that the number of ND cases will double by the year of 2050 . With treatments only available for symptom management and relief, disease prevention may yield significant benefits. Recently, there had been association drawn between the disruption of phospholipid (PL) homeostasis and the progression of NDs. Pathological developments were observed in cellular processes including autophagy, maintenance of mitochondrial integrity, and management of tissue oxidative stress. As PLs actively participate in the regulation of these cellular pathways and in neuronal signal transduction for the maintenance of an optimally functioning nervous system, their homeostasis is tightly controlled via an intricate system of interconversion and metabolism. Therefore, in this review, the contribution of a homeostatic PL pool and the detrimental effects by the lack thereof, are discussed in detail as it relates to ND development.
\end{abstract}

\section{Keywords}

Phospholipids; Kennedy pathway; neurodegeneration

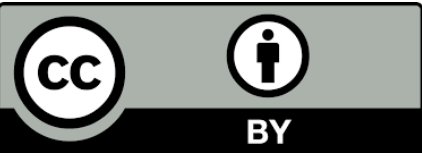

(C) 2021 by the author. This is an open access article distributed under the conditions of the Creative Commons by Attribution License, which permits unrestricted use, distribution, and reproduction in any medium or format, provided the original work is correctly cited. 


\section{Introduction}

The plasma membranes (PMs) are permeable barriers comprised of phospholipids (PLs), that separates the extracellular and intracellular compartments [1]. The major membrane PLs are phosphatidylcholine (PC) and phosphatidylethanolamine (PE), followed by phosphatidylserine (PS) and phosphatidylinositol (PI) [1]. As PLs make up 75\% of total lipid content, they play a central role in maintaining membrane structural integrity and function [2]. PLs are regulators of membrane fission and fusion events, cell division, apoptosis, phagocytosis, and many other critical functions [2, $3]$. The essentiality of membrane PL is evident in the animal models where embryonic lethality was observed consequential to the PL gene deletions and mutations. For example, complete disruptions of PE synthesis pathways are embryonically lethal in mice $[4,5]$. In humans, PE plasmalogen deficiency as a result of insufficient synthesis is a hallmark of peroxisomal disorders such as the Zellweger syndrome [6]. Recently reported are the involvements of PLs in illnesses in the central nervous system (CNS), including the disruption of choline transport via choline transporter-like protein 1 (CTL1) causing a novel childhood-onset neurodegenerative disease (ND) [7], and a disrupted PE biosynthesis pathway in PCYT2 and SELENO1 (EPT) mutant carriers causing hereditary spastic paraplegia [8, 9]. Additionally, recently reviewed by Rickman et al. are the functional lipid metabolic pathways in motor neuron diseases (MNDs) [10]. Cunnane et al. further discussed the current state of the art therapeutic strategies in treating NDs by targeting brain bioenergetics rescue, specifically in glucose, ketone, and TG metabolisms with affected cellular processes including mitochondrial respiration and health, insulin sensitivity, and hormonal balance. It was shown that the brain energy gap between sufficient ATP synthesis and cellular requirement is correlated with the development of ND and regulated by the neuroendocrine system [11]. This relationship between the brain energetics and NDs solidifies the notion that brain health is closely related to whole body metabolism.

NDs are a diverse group of neuropathological diseases that are currently incurable due to the irreversible neuronal loss [12] Development of neurodegenerative diseases are characterized by cell death and had often been associated with dysregulation of metabolism, mitochondrial dysfunction, accumulation of protein aggregates, and neuroinflammation, especially in the case of pathological protein aggregates: Parkinson's disease is associated with $\alpha$-synuclein aggregates [13], Alzheimer's disease with extracellular amyloid- $\beta$ plagues and cytosolic tau tangles [14], and Huntington's disease with hungtintin aggregates [15]. Although therapies are available for symptom management and relief the incurable state of these diseases warrants development of new treatments targeting the diseases on a molecular level, to prevent and potentially reverse the neuronal damages.

Recently, the association between membrane phospholipids and neurodegeneration had been implicated. As neurodegeneration is age-related, prevention is arguably one of the most important aspects in targeting these diseases as a public health issue [16]. As related to aging, the CNS experience deterioration in both functions and morphology [12] It had been projected that at the current rate of growth, the neuronal diseases will double by the year of 2050, with Lewy bodies 
dementia (LBD) being the most prevalent [17], therefore with rapid growth of the population affected by neurodegeneration, prevention for such diseases may yield significant benefits.

In this review, the major classes of phospholipids in the central nervous system (CNS) and the regulation of their homeostasis will be discussed, followed by the pathological development of neurodegeneration as attributed to the dysregulation of phospholipid homeostasis. Lastly, available therapies to treat lipid metabolic dysregulation are summarized to provide a comprehensive outlook of the current state of knowledge in neuronal PLs (Figure 1).

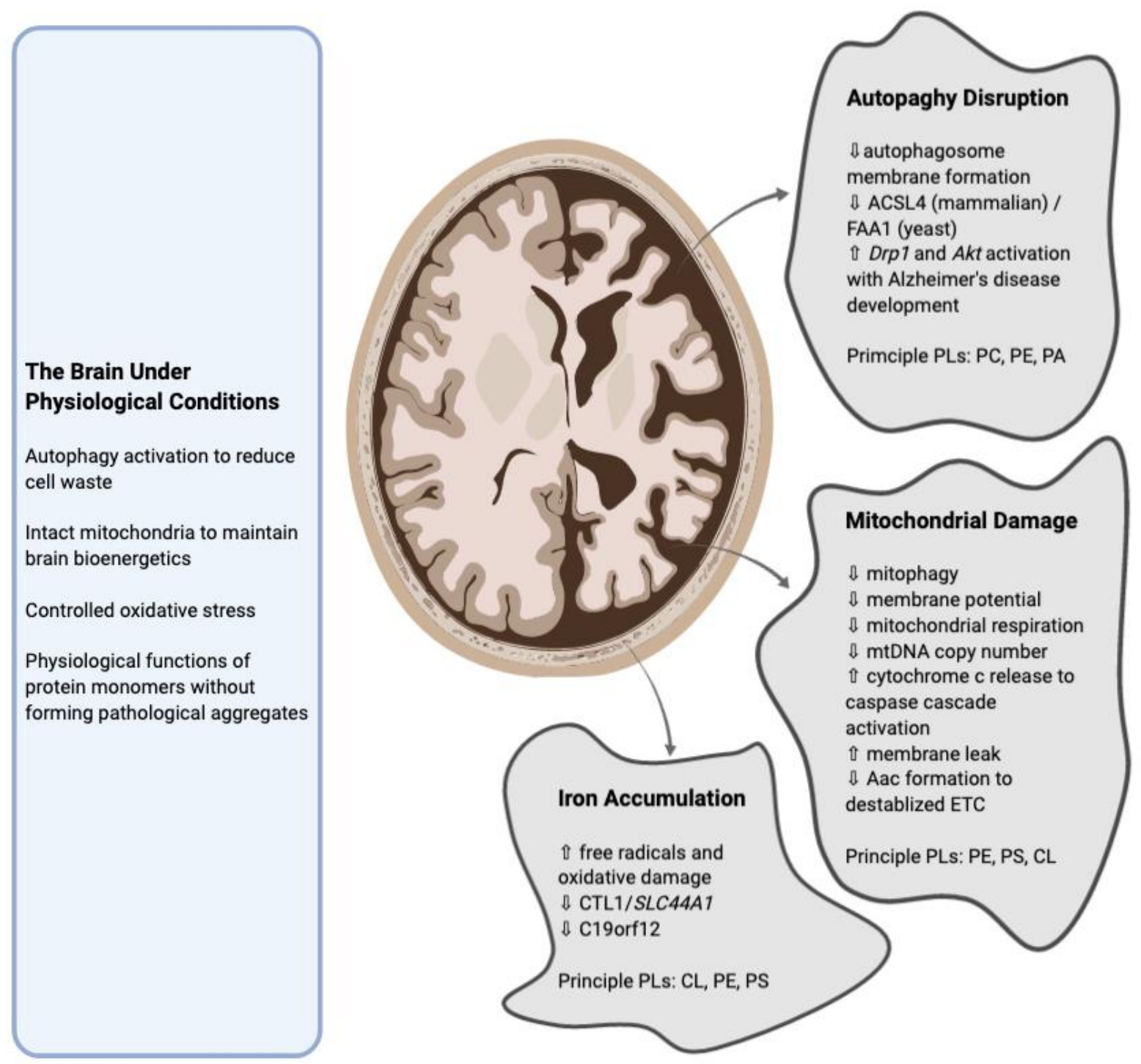

Figure 1 An illustration of pathological developments in cellular processes in Neurodegeneration, and the main phospholipids involved (Image generated with BioRender).

\section{Classes of Major Membrane Phospholipids}

Phospholipids are a broad family of amphipathic molecules composed of a glycerol backbone, a polar headgroup bound through a phosphate group at the sn-3 position, and two fatty acid ester bonds at the sn-1 and sn-2 positions $[18,19]$. The inclusion of both the hydrophilic and hydrophobic 
ends allows PLs to spontaneously form bilayer structures, with the hydrophobic fatty acids crowding the centre of the bilayer and the polar headgroups defining the general membrane architecture [1, 20]. PC and PE are the principal bilayer-forming phospholipids. PC contains a choline headgroup, a quaternary ammonium cation and PC typically localizes in the outside layer of the membrane. On the other hand, the ethanolamine headgroup on PE is smaller and gives the PE molecule a conical shape, as the fatty acid tails spread out wider with less force exerted from the headgroup [18]. As a result, $\mathrm{PE}$ is typically located in the inner membrane, and it maintains the concave curvature of the membrane. PE shares the inner membrane layer mainly with PS, with the only exception being in the mitochondria where cardiolipin $(C L)$ is the second major PL $[2,21,22]$. Because of its serine headgroup, PS carries a negative charge and contribute in the formation and maintenance of the membrane electrochemical gradient [21].

\subsection{Phosphatidylcholine Biosynthesis}

PC biosynthesis predominantly occurs via the CDP-choline arm of the Kennedy pathway, by the liver PE methylation pathway (PEMT), and by PC remodeling by the Lands cycle [18, 23]. The CDPcholine pathway shares the enzymes and substrates with to the CDP-ethanolamine arm od the Kennedy pathway for PE synthesis (Figure 2), which are the only de novo biosynthesis pathways for PC and PE [24, 25]. The CDP-Cho Kennedy pathway utilizes exogenous choline and DAG. Choline is quickly phosphorylated by choline kinase (CK), forming phosphocholine. Then, through the action of CDP-choline cytidylyltransferase (CT/Pcyt1) using CTP as a substrate, phosphocholine is converted to CDP-choline, a high-energy intermediate. The CT/Pcyt1 step is the rate-limiting step in de novo PC synthesis. Lastly, the phosphocholine headgroup is transferred onto diacyglyerol (DAG) by CDP-choline:1,2-diacylglycerol choline phosphotransferase (CEPT) to form the final PC product [24]. The Kennedy pathways specificity is determined by the regulatory enzymes, CT/Pcyt1 and EC/Pcyt2 that exhibit strong substrate specificity [26]. 


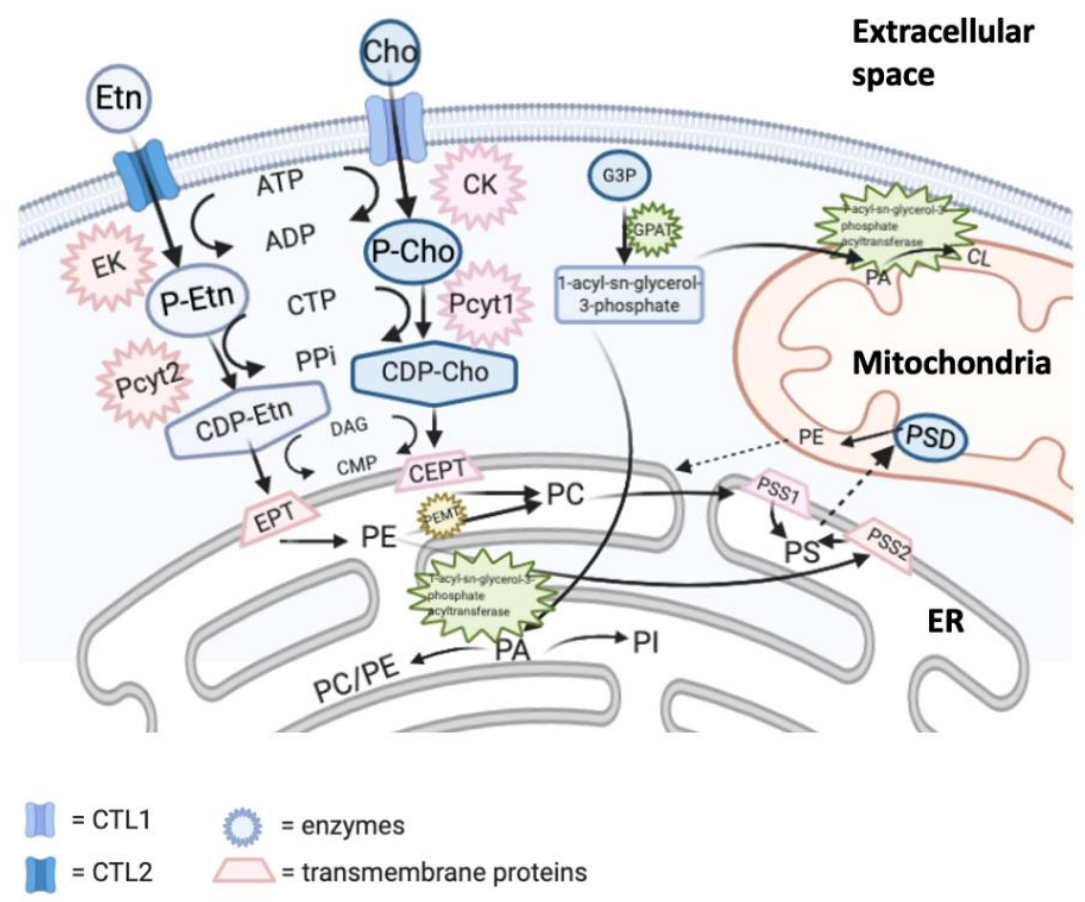

Figure 2 Biosynthesis pathways for the major phospholipids. PE and PC can be synthesized de novo via the CDP-choline/ethanolamine (Kennedy pathway) using exogenous choline and ethanolamine. Interconversion occurs between synthesized PLs between the ER, the MAM, and the mitochondrial inner and outer membranes. Etn ethanolamine, Cho choline, EK ethanolamine kinase, CK choline kinase, Pcyt2 CDPethanolamine cytidylyltransferase, Pcyt1 CDP-choline cytidylyltransferase, P-Etn phosphoethanolamine, P-Cho phosphocholine, EPT DAG:CDP-Etn phosphotransferase, CEPT CDP-choline:1,2-diacylglycerol choline phosphotransferase, PEMT Phosphatidylethanolamine N-methyltransferase, G3P_glycerol-3-phosphate, GPAT G3P acyltransferase, PSS1 CDP-diacylglycerol--serine O-phosphatidyltransferase 1, CDPdiacylglycerol--serine O-phosphatidyltransferase 2, PSD phosphatidylserine decarboxylase, PC phosphatidylcholine, PE phosphatidylethanolamine, PA phosphatidic acid, PI phosphatidylinositol, PS phosphatidylserine, CL cardiolipin. Image generated with BioRender.

The regulation of the CDP-Cho Kennedy pathway is predominantly at the level of CT/Pcyt1 but substrate availability could contribute [27]. There are two isoforms of Pcyt1, $\alpha$ and $\beta$, and they are encoded by separate genes. Pcyt1 $\alpha$ had been extensively investigated and most recently reviewed [28]. Interestingly, Pcyt1 $\beta$ is abundantly expressed in neuronal cells, specifically in the distal axons of primary neurons $[24,29]$. However, both Pcyt1 $\alpha$ and $\beta$ isoforms have been demonstrated to have increased expression upon neuronal cell differentiation [30]. This close association between neuronal cell differentiation and PC synthesis is further extended to CEPT activity, where nerve growth factor (NGF) induced PC synthesis via activation of the CEPT protein [31]. Collectively, these results indicate a significant role PC plays in neuronal cell proliferation. 
Another pathway for PC synthesis is the liver specific PEMT pathway. This pathway accounts for $30 \%$ of hepatic PC production and is the main source of choline when demands for choline are high such as during the pregnancy and lactation [25, 32]. A total of three methylation reactions with the methyl group donor S adenosylmethionine (SAM) are involved in the conversion of PE to PC [33]. Lastly, PC can also be produced via the Lands cycle, where the sn-2 fatty acid can be inserted onto the glycerol backbone of a lyso-PC molecule by lysophosphatidylcholine acyltransferases (LPCATs) activities. There are currently four identified LPCATs, and their activities in using fatty acids as a substrate can modulate TAG storage in a different manner than by the Kennedy pathway [23].

\subsection{Phosphatidylethanolamine Biosynthesis}

There are three pathways for the biosynthesis of PE in mammalian cells. The predominant pathway is the CDP-ethanolamine pathway (Kennedy) pathway, to produce PE from ethanolamine and DAG (Figure 2). The second pathway is the PS-decarboxylation (PSD) pathway, which specifically occurs in the mitochondria. The third pathway is the FA remodeling Lands pathway [34]. Recent studies had shed light on the significance of a PE synthesis in mice and humans. A complete knockout of murine Pcyt2 is embryonically lethal [4]. Disruption of the PSD pathway in Pisd knockout mice is also embryonically lethal [5], demonstrating that both the microsomal Kennedy pathway and the PSD mitochondrial pathway produce different, yet developmentally essential pools of PE. Furthermore, mutations in Pisd had been linked to diseases including the multi-organ disease of Liberfarb syndrome, a spondyloepi-metaphyseal dysplasia with mitochondrial damage, and cataracts, skeletal dysplasia, and white matter alterations [35]. These results further indicate the essential role PSD plays in maintaining the mitochondrial PE pool. Finally, similar to PC, PE membrane diversity is further achieved via the Lands cycle where the PLs are modified through deacylation and reacylation reactions [33]. Additional to the LPCATs, two mammalian lysophosphatidylethanolamine acyltransferases (LPEATS) had also been identified. Specifically, LPEAT1 is preferentially active towards 18:1-CoA, whereas LPEAT2 prefers 18:1-CoA or 20:4-CoA. LPCAT3 and LPCAT4 also have LPEAT activity and with a preference for polyunsaturated CoA [36].

\subsubsection{The CDP-Ethanolamine Pathway}

The CDP-ethanolamine arm of the Kennedy pathway (Figure 2) is the only de novo synthesis pathway for PE. Through a series of three reactions, this pathway incorporates the exogenous ethanolamine into a DAG backbone. In the first step, ethanolamine (Etn) is phosphorylated by ethanolamine kinase, through which phosphoethanolamine (PEtn) is formed. CDP-ethanolamine cytidylyltransferase (ET/Pcyt2) then catalyzes the rate-limiting reaction in which PEtn is converted into CDP ethanolamine (CDP-Etn). Lastly, DAG:CDP-Etn phosphotransferase (EPT/SELENO1) condenses PEtn and DAG, producing the final PE product [33]. The importance of the CDP-Etn pathway and the key enzyme (ET/Pcyt2) in general lipid metabolism was established in the Pcyt2//+ heterozygous mice (ETKO) when we showed that those mice develop a surprising phenotype similar to human metabolic syndrome, including hypertriglyceridemia, liver steatosis, insulin resistance and obesity [4]. In addition to the metabolic syndrome, the Pcyt2 deficiency caused a sexual dimorphism with male but not female ETKO developing hypertension and cardiac hypertrophy [37]. Excess toxic DAG unused in the CDP-ethanolamine pathway requires an increased formation of fatty acids by lipogenesis to produce neutral triglyceride, but consequently developing into metabolic syndrome 
in older ETKO [38]. A similar triglyceride (fat) accumulation was subsequently shown in the liver specific Pcyt2 knockout mice confirming the DAG hypothesis [39]. Muscle-specific Pcyt2 knockout mice however had elevated DAG content but did not develop insulin resistance [40], which was opposite from ETKO, indicating a multiple organ requirement for development of metabolic disease, as well as a specific role for ET/CDP-ethanolamine pathway in the skeletal muscle.

We functionally characterized three ET/Pcyt2 protein isoforms: Pcyt2 $\alpha,-\beta$, and $-\gamma$. They are products of different splicing mechanisms of a single Pcyt2 gene [41]. The Pcyt2 $\alpha$ is the longest form and Pcyt $2 \beta$ is produced by an evolutionary conserved "exon-skipping" mechanism of exon 7. Pcyt $2 \beta$ is missing the central 18-amino acid linker region, causing the two isoforms to differ in the enzyme activity and regulation by phosphorylation. Pcyt2 $\gamma$ is a product of an "intron-retention" splicing mechanism as a result of an alternative usage of $5^{\prime}$ donor and $3^{\prime}$ acceptor sites [42, 43]. Pcyt2 $\gamma$ contains the central linker region as Pcyt $2 \alpha$ but lacks the second cytidylyltransferase domain at the C-terminus, rendering it enzymatically inactive [42]. Pcyt2 $\alpha$ and Pcyt2 $\beta$ are catalytically active as homo- and heterodimers [43]. Pcyt $2 \gamma$ can dimerize and inhibit Pcyt $2 \alpha / \beta$ activity, demonstrating its role as a natural suppressor of the Kennedy pathway. A further mutation analysis showed that the highly conserved CTP binding HXGH motifs in the N- and C- Pcyt2 catalytic domains are required for the full enzymatic activity [42]. Indeed, a recently reported human mutation in the C-terminal HXGH completely disrupts Pcyt2 activity and the CDP-Etn Kennedy pathway, resulting in complex hereditary spastic paraplegia [9].

\subsubsection{The PS-Decarboxylation Pathway}

Mitochondrial PE is produced via PS decarboxylation by PS decarboxylase (PSD/Pisd) at the inner mitochondrial membranes (IMM) [3, 44]. PSD pathway produces different PE species from the Kennedy pathway, including significantly more PUFA-containing PE [45]. PE produced by PSD pathway is mostly retained in the mitochondria, yet some PE could be transported to the ER. The transport specificity is associated with the side-chain saturation status of the PE: specifically, 32:2, 34:2 and 38:6 PE species are preferentially exported from the IMM while 38:4 is largely retained in the mitochondria [45]. The PSD protein is a product of a single gene Pisd; the protein precursor peptide encoded by Pisd is cleaved into separate $\alpha$ and $\beta$ subunits which then form active PSD heterodimers [46]. Whereas the mammalian PSD resides strictly at the IMM, yeast PSD1p exhibits both mitochondrial and ER localization that produce different PE pools from the de novo Kennedy pathway [47]. According to Human Protein Atlas human PSD strictly reside in the mitochondria but mammalian PSD needs further studies for full characterization.

\subsection{Phosphatidylserine Biosynthesis}

In mammalian cells, PS cannot be produced via de novo synthesis and is exclusively produced from pre-existing PLs: PC and PE. PS synthase 1 (PSS1/Ptdss1) and PS synthase 2 (PSS2/Ptdss2) perform $\mathrm{Ca}^{2+-}$ dependent headgroup-exchange reactions to convert PC and PE into PS, respectively $[34,48,49]$. The two enzymes reside in the ER membranes but most abundantly in the ER-MAM where PS synthesis and many other important lipid related processes occur [50]. PSS1 and PSS2 exhibit low similarity and although ubiquitously present, their expressions pattern implicates some tissue specificity [49]. PSS2 is the most abundant in the testes while PSS1 is more expressed in the kidney, brain, and liver $[48,51]$. PSS1 is limiting for the overall PS synthesis, as demonstrated by 
overexpression of PSS1 but not PSS2 in cell culture models [49, 52]. Compared to PSS1, PSS2 produces more PUFA-containing PS and is under stronger 'end-product' inhibitory regulation [53]. PS binds PSS1 at Arg-95 and PSS2 at Arg-97 and mutations into either of these sites inhibits the enzyme activity $[54,55]$. Moreover, the gain of function mutation in the PS-binding region of PSS1 causes the Lenz-Majewski syndrome (LMS). The most prominent symptoms in patients with LMS are hyperostosis, intellectual impairment, and dwarfism. As the authors noted, no known association had been previously reported between PS and skeletal dysplasia; it was hypothesized the resultant outcome could be attributed to PS's ability in binding calcium, thus the lack thereof may disrupt bone calcification [56].

\subsection{Phosphatidic Acid, Cardiolipin and Phosphatidylinositol Biosynthesis}

PA is a minor PL group that is centrally situated in the synthesis of major PLs. PA is converted to CDP-DAG by the CDP-DAG synthase for the syntheses of $\mathrm{CL}$ and $\mathrm{PI}$, or it may be converted to DAG by PA phosphatases for the syntheses of PC and PE [25]. Therefore, PA metabolism is tightly regulated via multiple mechanisms including protein phosphorylation, membrane association, and other components of lipid metabolism such as the end-product availability $[25,57]$.

PA synthesis begins with the conversion of glycerol-3-phosphate (G3P) to 1-acyl-sn-glycerol-3phosphate via acylation by G3P acyltransferase (GPAT). Then, the second acylation catalyzed by 1acyl-sn-glycerol-3-phosphate acyltransferase, which produces PA [22]. At least four mammalian GPAT isoforms had been identified that are products of different genes; GPAT1 and GPAT2 are predominantly expressed in the mitochondria, whereas GPAT3 and GPAT4 are ER-bound [22]. PA can be resynthesized from $P C$ and $P E$ by the action of phospholipses, particularly phospholipase $D$ [25]. GPATs exhibit some substrate preference, with GPAT1 having a higher preference for saturated FAs. GPAT4 increased PE and PI synthesis, while altering the PC level as well. Moreover, GPAT isoforms also catalyze TAG synthesis [58]. These four, and potentially more, GPAT isoforms function in an intricate system to balance PA/DAG distribution between PL and TAG and therefore between membrane function and lipid storage.

The main mitochondrial PLs cardiolipin (CL) is synthesized from PA as a precursor. In CL synthesis, PA is converted with CTP to the high energy intermediate, CDP-DAG, in the rate limiting step, catalyzed by cytidinediphosphate-1,2-diacyl-sn-glycerol (CDP-DAG) synthetase (CDS) [22]. In the mammalian genome, three proteins can function as CDS; namely, they are CDS1, CDS2, and TAMM4. CDS1 and CDS2 both reside are the ER membrane while TAMM41 is bound to the IMM [59]. The CDP-DAG formation is slow and requires the translocation of PA from the outer layer of the OMM into the IMM where the condensation reaction occurs [22]. First, phosphatidyl group is transferred from CDP-DAG to G3P as catalyzed by PGP synthase (Pgs1), forming phosphatidyl-glycerophosphate (PGP). Subsequently, phosphatidylglycerol (PG) is produced via tyrosine phosphatase mitochondrion 1 (PTPMT1) catalysed hydrolysis of PGP, and the last step of CL synthesis involves the transfer of a second activated phosphatidyl group from CDP-DAG by CL synthase activities [22].

$\mathrm{CL}$ has stringent acyl group specificity, therefore, remodelling and resynthesis of the $\mathrm{CL}$ species is critical, provided the lack of acyl specificity in the de novo CL synthesis pathway. The two remodelling pathways are the Lands cycle where a two-step acylation-reacylation occur, and the direct acyl group exchange between PLs [60]. Tafazzin is a phospholipid-lysophospholipid transacylase with the ability to catalyze $\mathrm{CL}$ remodelling in both pathways. In both pathways for $\mathrm{CL}$ 
remodelling, tafazzin as a transacylase catalyzes the production of a remodeled CL and a monolyso$\mathrm{PL}$ from a monolyso-CL and a PL. Interestingly, tafazzin-mediated $\mathrm{CL}$ remodelling generates a shift towards unsaturation in the acyl chains which is critical for membrane fluidity [60]. In addition, Monolyso-CL acyltransferase 1 (MLCLAT1) and acyl-CoA:lysocardiolipin acyltransferase-1 (ALCAT1) are both enzymes functioning in the process of $C L$ remodelling and utilize acyl-CoA as the acyl donor [60].

The other possible metabolic fate for PA as a precursor is the de novo synthesis of PI. In this pathway, PA is converted to CDP-DAG by CDS1 and CDS2 at the ER membrane without being transported into the mitochondria [59]. While PI is highly enriched in stearic acid at the sn-1 position, and arachidonic acid at the sn-2 position, there is limited research and understanding of whether the CDS's have acyl chain specificity. This acyl chain specificity of $\mathrm{Pl}$, however, had been demonstrated to be tissue-specific, as it is present in $>85 \%$ of the brain PI pool $[59,61]$. As well, CDS activities are essential for PI synthesis. The last step in the pathway is catalyzed by CDPdiacylglycerol-inositol 3-phosphatidyltransferase (CDIPT), also called PI synthase (PIS). This reaction occurs via exchanges of inositol and CMP on CDP-GAD, thus producing PI [61]. Remodelling of PI post de novo synthesis is an additional step for the PI species to achieve a desired acyl group composition [62].

\section{Membrane Phospholipids in the Central Nervous System}

\subsection{The PL Specificity of the Nervous System}

As essential components of neuronal membranes PLs are responsible for proper functioning of the synapses, receptors, transporters, neurotransmitters and lipid-signaling. PLs are in a constant turnover through highly regulated processes of synthesis and degradation. They are source of various signalling molecules including eicosanoids, fatty acids, PA, and DAG. PE is the main provider of $n-3$ and $n-6$ polyunsaturated fatty acids (PUFA) and monounsaturated fatty acids (MUFA), and it is also the precursor of the specific neurotransmitter anandamide ( $\mathrm{N}$-arachidonoyl ethanolamine) $[63,64]$. PC is also a source of choline for acethylcholine synthesis, and PI is the precursor of multiple phosphoinositide species with a wide functional diversity in the CNS [24, 62].

Recent studies revealed a large diversity in the brain PL species, compared to other organs. There is a larger cholesterol and a lower PC content in the human brain than in average bodily tissue [10, 63]. Moreover, compared to non-brain tissues, higher FA saturation, together with high cholesterol is necessary for optimal membrane function in the brain [63]. A recent review on the lipidomic analyses of the brain PL content also highlighted that PLs in the brain are more saturated than in other tissues, further elucidates the importance of PL homeostasis for maintaining the proper brain function [63]. In support of this, computational modeling of the mammalian brain cell plasma membrane also showed a higher tail order as a result of high saturation and cholesterol density, especially in the outer layer, as a specificity of the PL bilayer in the brain [65]. However, because of the high PLs diversity and complexity of regulation, development of new approaches is needed in studying the brain lipids, as the need for better biological models for neurobehavioral studies have already been recognized. 


\subsection{The Critical Regulators of PLs Synthesis in the Nervous System}

Although constantly remodeled, the stable membrane PL ratio is at the centre of optimal cellular function and its disturbance is frequently of pathological nature. For example, an inversed hippocampal PC/LPE to PS ratio is indicative of postischemic neurodegeneration in the rat CNS [66]. The PL composition in the mouse brain mitochondria differs by age, and the PL content deteriorates overtime [67]. PC/LPC ratios is significantly different in patients with Alzheimer's disease (AD) and mild cognitive impairment [68], and an altered PL composition was also observed in the brains of patients with Down syndrome [69].

An intricate set of regulation ensures the optimal PLs synthesis in neuronal membranes. Choline transporter-like protein 1 (CTL1) encoded by the SLC44A1 gene is the main transporter for the brain PC synthesis, while a different transporter ( $\mathrm{CHT} / S L C 5 A 7)$ regulates choline transport for acetylcholine synthesis [70]. Recent studies had shown that both SLC44A1-PRKCA and NOTCH1PRKCA fusion oncogenes are causing development of papillary glioneuronal tumors [71]. CTL1 activity is regulated by choline availability, evidenced by a decreased expression in choline deficiency and an increased expression by choline supplementation [27]. Homozygous deletion of CTL1 blocks choline transport and disturbs PL homeostasis by reducing PE and PS pools as means to preserve the brain PC as a source of choline [7]. Recently, we identified a new role for CTL1/SLC44A1 and CTL2/SLC44A2 proteins in transporting ethanolamine for PE synthesis. Therefore, since CTL1/2 transport plasma membrane choline and ethanolamine, they directly control PC and PE homeostasis and their membrane ratio [72]. Both CTL1 and CTL2 additionally localize and transport choline and ethanolamine in the mitochondria yet their role in the mitochondria is not completely clear [72].

Our search of the Ensemble Database showed that Pcyt2 splice variants similar to mouse Pcyt2 $\gamma$ (section II. b) i)) are present in humans and distantly related species, indicating evolutionary conservation of splicing mechanisms to produce multiple transcript variants. Indeed, multiple human transcripts had been identified that lack HXGH catalytic site as we demonstrated for mouse Pcyt2; examples include ENST00000570388.5, ENST00000331285.7, and ENST00000572995.5, all of which lack the C-terminal $\mathrm{HXGH}$ site. In addition, Siberian tiger Pcyt2 transcript (ENSPTIG00000010590) lacks the C-domain HXGH, gorilla variant (ENSGG0G00000067604.1) has the N-domain $\mathrm{HXGH}$ absent; zebrafish has several variants lacking either of the two HXGH catalytic sites. Excitingly, the most recent study in Drosophila established that dysfunctional minor spliceosome causes spinal muscular atrophy by direct contribution from three neural genes, Pcyt2, Zmynd10, and Fas3 as the main splicing targets [73]. This agreed with the existence of multiple Pcyt2 isoforms and implicates a strong splicing and self-inhibiting regulation of de novo PE synthesis by the Kennedy pathway. However, whether is such mechanism fully active in the human CNS is not known at the present.

Pcyt2 expression and activity are suppressed by oxysterols and stimulated by early growth response factors, nutrient starvation and PKC signaling. Oxysterols is a potent cholesterol regulator that stimulates the excretion of cholesterol. It was also shown to inhibit Pcyt2 [74, 75], specifically by promoter interaction of NF-Y and $\mathrm{p} 300$ and histone $\mathrm{H} 3 \mathrm{~K} 27$ acetylation [76]. Oxysterols have been implicated in HD, AD, ALS, and PD [77]; therefore, down regulation of Pcyt2, PE synthesis, and $\mathrm{PE} /$ cholesterol homeostasis by oxysterols warrants further investigation in the CNS. 
The metabolic conversion of PC, PE and PS are tightly intertwined (Figure 2): PC and PE universally produce PS by PSS1 and PSS2, respectively; the mitochondria produce PE from PS by PSD; and mainly, the liver PE could be converted to PC by the PE methyltransferase (PEMT) [25].

Since there are two pathways for PE synthesis, the activities of the CDP-Etn Kennedy pathway gene Pcyt2 and PSD pathway Pisd gene are coordinately regulated. Pis $d^{+/-}$mice has increased Pcyt2 mRNA and an increased Kennedy pathway activity [5]. On the other hand, excess Etn up-regulates the PE Kennedy pathway and reduces the export of PE derived in the mitochondria [45]. PE produced via PSD pathway resides in the mitochondria, but it could also be exported into the ER when the PE Kennedy pathway is impaired [45], as in the case of liver specific Pcyt2 knockout mice [39]. Neither pathway, however, can fully compensate for the other as the global mouse knockouts of Pisd or Pcyt2 are both embryonically lethal [4, 5].

A sexual dimorphism for the PE formation in the mitochondria had been observed in the hepatocytes of $\mathrm{Pss}^{-/}$mice. Hepatocytes from female Pss2 $2^{--}$mice showed increased choline incorporation in PC produced via CDP-choline Kennedy pathway by increased Pcyt1 activity, whereas hepatocytes from male $P s s 2^{-/}$mice showed increased ethanolamine incorporation into PE and PC via Pcyt2 (CDP-Etn Kennedy pathway) and PE methylation (PEMT) activities, respectively [78]. Provided that $\mathrm{Pss}^{-/}$cells need to rely primarily on PSS1 for the synthesis of PS, it was evident that female mice relied more heavily on the de novo synthesized PC to form PS and maintain PL homeostasis. On the other hand, males rely more heavily on the de novo synthesis of $\mathrm{PE}$, and potentially PEMT for PC to maintain phospholipid homeostasis [20]. This is in support of the sexual dimorphism observed in whole-body Pcyt2 knockdown model, in which male mice were more severely impacted by the disruption of the rate limiting PCYT2 in the Kennedy pathway [37].

Finally, the existence of the human brain PE methylation is a controversial issue. A reduced PEMT activity via a functional single nucleotide polymorphism, G523A (the rs7946 allele), in a Chinese Han population showed correlation with sporadic $A D$, especially in females. No significant alterations were observed in homocysteine production by the mutated PEMT; therefore, it was speculated an altered PC production could be causal [79]. Our recent work with individuals that develop a new type of childhood neurodegeneration showed an CTL1 mutant fibroblasts increased PC formation from PE methylation, which could be important for future treatments if proved that same is the case in the patients' neurons or stem-cell derived neurons [7].

\section{Membrane Phospholipids in Neuropathology}

As previously discussed, the enzymes involved in the regulation of phospholipids function in multiple cellular pathways to ensure a homeostatic environment for the neural cells. At large, the major pathways implicated in neuropathy because of PL dysregulation include disruption in autophagy \& endocytosis, mitochondrial damage, and iron accumulation \& oxidative stress because of other organelle damages.

\subsection{Autophagy \& Endocytosis (Lysosomes)}

Autophagy and the ubiquitin-proteasome system (UPS) are the two cellular processes to recycle cellular materials and eliminate deleterious cytosolic content by lysosomal degradation [80]. Three main types of autophagy exist in the mammalian cell differed by their mechanisms of actions: microautophagy, chaperon-mediated autophagy (CMA), and macroautophagy. Microautophagy is 
the unselective, direct engulfment of cytoplasmic cargo by lysosomes, and CMA is through binding of lysosome-targeted heat shock proteins to selected cytoplasmic substances [81, 82]. The most well-studied type of autophagy is macroautophagy, where a series of double-membraned intracellular complexes are formed in the cytosolic compartment that eventually fuse with lysosomes for degradation [80, 83]. The macroautophagic process and the UPS system work in concert with one another, and both are implicated in the maintenance of the physiological integrity of the CNS [80, 84]. Macroautophagy is hereafter simply referred to as autography, a cellular process initiated by the unc-51-like autophagy-activating kinase 1 (ULK1) complex and negatively regulated by rapamycin complex 1 (mTORC1). Upon the initiation of autophagy, a cup-shaped structure with a lipid bilayer membrane called phagophore is nucleated near the cytosolic content to be cleared from the cell. This cup-shaped structure then undergoes expansion in the elongation stage by membrane addition [85].

Previously, it was generally agreed upon the lipid flux into autophagophores in both mammalian and yeast cells are contributed by preformed membranes from a combination of the endoplasmic reticulum (ER), the Golgi apparatus, endosomes, mitochondria, and even the plasma membrane itself. Contradictory to this hypothesis, Schütter et al. recently reported in yeast cells that localized $\mathrm{PL}$ synthesis as mediated by acyl-CoA synthesase 1 (FAA1) is imperative for autophagophore expansion and autophagosome formation [86]. Additionally, similar mechanisms had been reported in mammalian cells, where the de novo PL synthesis is required for autophagosome membrane formation. Our laboratory first demonstrated that the de novo synthesis of PLs at the ER membrane is coupled with autophagophore formation and that PE made by the Kennedy pathway incorporates into LC3 [87]. Recent findings by Andrejeva et al. confirms this and further elucidates the de novo synthesis of $P C$ is required for autophagosome formation and maintenance. Specifically, the newly synthesized PC contains a higher polyunsaturated FA content and is directly incorporated into autophagophores [88]. Indeed, when expressed in yeast cells, the human long-chain-fatty-acid CoA ligase 4 (ACSL4) protein was able to replace yeast FAA1 activity and carry the downstream formation of autophagosome [86].

In the CNS, autophagy is a main pathway for drug target for the treatment of neurogenerative diseases. Amyloid- $\beta$ treated human neuroblastoma cells expressed sustained up-regulated levels of protein kinase B (Akt) by dynamin-related protein 1 (Drp1) activation, and inhibition of autophagy via the mTOR pathway [89]. The deleterious impact was observed in apoptosis of the Amyloid- $\beta$ treated cells. Interestingly, the effect of Akt activity in maintaining neuronal cell health is controversial: on one hand, Akt activation stimulates autophagy via inhibition of mTOR activities, and is central for maintaining the mitochondrial integrity [90]; on the other hand, Akt up-regulation has been associated with AD development, potentially via an overactive UPS induced by prolonged Akt activation [89, 91]. In either case, Akt remains an important regulator for neuronal cell survival. Recently, it was reported the lipid-sensing ability of mTOR as both mTORC1 and mTORC2 are activated upon de novo PA synthesis of 16:0 and 18:1 PA species with subsequent Akt phosphorylation, suggesting a possible avenue of PA synthesis modulating autophagy and the neuronal cell fates [92]. 


\subsection{Mitochondrial Damage}

The mitochondria are cellular organelles of prokaryotic origins, with features including PL-bilayer membranes and its own set of circular genome (mtDNA), separate from the chromosomal DNA. The incorporation of both the outer and inner mitochondrial membranes (the OMM and IMM) in the mitochondria allows the buildup of an electrochemical gradient within the intramembrane space, for substrate transport and energy production through the electron transport chain (ETC). The physiological significance of the mitochondria is largely derived from its ability to generate ATP, a high-energy molecule essential to fuel cellular processes via oxidative phosphorylation (OXPHOS). The production of reactive oxygen species (ROS) is a by-product of OXPHOS and despite its role as a secondary messenger, ROS cause oxidative stress when overproduced [93].

The mitochondria membrane integrity is governed by fusion and fission events, mtDNA replication, and the level of oxidative stress. Particularly, fusion and fission events are crucial for the mitochondria quality control (MQC) [93]. Fusion events are mediated by core enzymes of mitofusin1(Mfn1) and mitofusin2 (Mfn2) for OMM fusion, and optic atrophy gene 1 (OPA1) for IMM fusion [94]. Fusion redistributes mitochondrial content in the presence of mild and temporary stressors to "dilute" the damage of partially dysfunctional areas. Hyperfusion may even occur in the case of nutrient starvation to combat a complete breakdown of the mitochondria [93, 95]. Contrarily, fission events in the mitochondria is the process of organelle fragmentation into separate entities. Fission is mediated largely by the dynamin-related protein 1 (Drp1), where the irreversibly damaged mitochondria undergo mitophagy, a selective form of autophagy targeted at the mitochondria [96].

PTEN-induced putative kinase 1 (PINK1) is a central activator of mitophagy and MQC. The accumulation of PINK1 on the OMM activates two pathways: 1) PINK1 can recruit Drp1 to mitochondria to facilitate mitochondrial fission, and 2) PINK1 can complex with Parkin which recruits ubiquitin, thus inducing mitophagy. The PINK1-Parkin system is inhibitory of mitochondrial fusion by downregulation of $\mathrm{Mfn} 1$ and $\mathrm{Mfn} 2$, selectively pinching off fragmented mitochondria [95]. Recent data also demonstrates a PINK1-Parkin-independent pathway of mitophagy, facilitated by AMPK's activity in phosphorylating mitochondrial fission factor (MFF). ULK1 phosphorylation is also observed with induced AMPK activity in a dose-dependent manner. This newly discovered pathway suggests a more complex regulation of mitochondrial fission machinery with mitophagy, as ULK1 is a core protein in the ULK complex that is essential for autophagy initiation [97]. Interestingly, PINK1Parkin system could also promote mitochondrial biogenesis by facilitating degradation of a Ppargcoactivator 1-alpha (PGC-1 $\alpha$ ) inhibitor, PARIS [98]. In cortical neurons, overexpressed Parkin and PGC-1 $\alpha$ promoted mitochondrial biogenesis, respiration and mitophagy [99]. The induction of PINK1-Parkin with rotenone however inhibited PGC-1 $\alpha$ expression in PC12 cells [100]. Nevertheless, both studies demonstrated a bidirectional interaction between the PINK1-Parkin system and PGC$1 \alpha$ but the contradictory results from these studies warrants further investigation.

A balancing act of the membrane fusion and fission events via the sophisticated network dynamics is especially important in prevention of neurodegeneration, as mitochondria plays a crucial role in a high-energy demanding environment like in the nervous system. In fact, neuroinflammation and accumulation of toxic protein aggregates are implicated along mitochondrial damage in neuronal cells [101]. Dysregulated fusion and fission events are both deleterious in neuronal cells, since mitochondrial elongation and swelling due to hyperfusion, as well as fragmentation as a result of unchecked PINK1-Parkin activity and increased fission, can both 
lead to accumulation of damaged mitochondria and hence cell death [95, 102]. Moreover, the regulatory proteins discussed above had been implicated in different neurodegenerative diseases. To name a few, a malfunctioning PINK1-Parkin system via loss-of-function mutations is the leading cause for autosomal recessive Parkinson's disease; molecularly, the implication of the PINK1-Parkin system was demonstrated in a model of PARIS-dependent dopaminergic neuronal death by PINK1Parkin inactivation [98]; moreover, Drp1 can be activated by Amyloid- $\beta$ aggregates in the case of Alzheimer's disease (AD), as well as being a modulator in the process of neuronal differentiation by tempering the optimal level of fission event occurrence [89]. Aside from chronic neurodegenerative diseases, acute neurodegeneration such as traumatic brain injuries (TBI) also show altered mitochondrial fusion by up- and down- regulation of the OPA1 protein, depending on the grade of TBI [96].

The manifestation of mitochondrial damage occurs in various forms including oxidative stress, a reduced mitochondrial membrane potential $\left(\triangle \Psi_{\mathrm{m}}\right)$, mutation in mtDNA and/or a reduced copy number. PLs form autophagosome membranes, and protein LC3 which regulates autophagy is specifically lipidated with PE-hence regulating the mitochondrial and neuronal health [87]. In addition, prevention of apoptosis is an important avenue in combating the lack of cellular regeneration that may lead to degenerative diseases. Several PLS are heavily involved in the regulation of $\triangle \Psi_{\mathrm{m}}$, including $\mathrm{PE}$ and $\mathrm{CL}$. Specifically, reduced mitochondrial PE synthesis from PS had led to decreased level of mitochondrial respiration, along with disrupted IMM morphology and CL loss in muscle cells, despite the rescue efforts with the up-regulation of Parkin [103]. Furthermore, phosphoethanolamine (PEA), a substrate for PE synthesis, had been shown to disrupt mitochondrial respiration by indirect inhibition of the ETC protein complexes [104], more specifically by the inhibition of complex II [105].

With the metabolism of $\mathrm{mtPE}$ is closely regulated by the transport of PS, it also gives way to the indirect impact by the functions of the MAM and ER-mitochondria contact sites (MCSs) on mitochondrial vitality. Although no current consensus had been drawn on the mechanism of action of PS import to the IMM, the close juxtaposition of the MAM and mitochondrial membrane is needed for the PL transport to take place [34]. Mfn2 had undergone heavy investigation as an enzyme of interest in the tethering of the two membranes; however, there is no evidence of PS influx in relation to the mitochondrial fusion event. Whether the mitochondrial damage due to the loss of PSD was a result of intramitochondrial PS accumulation or loss of PE needs further investigation.

$\mathrm{CL}$ is a mitochondria-specific PL in the mammalian cell, which plays a central role in maintaining mitochondrial integrity. Nearly not present in any other cellular membranes outside the mitochondria, $\mathrm{CL}$ is the only PL with two phosphatidylglycerol backbones and four fatty acid side chains, rendering it another non-bilayer forming PL with a conical shape, aside from PE [106]. Due to its shape, $\mathrm{CL}$ is highly present in the IMM in an asymmetric manner across the lipid bilayer at sites with a high level of negative curvatures. The relation between a high concentration of $\mathrm{CL}$ and a curvature-dense environment was shown to be bidirectional, as in vitro induction of membrane $\mathrm{CL}$ accumulation by increasing the membrane curvature could be observed with only egg $\mathrm{PC}$ and $\mathrm{CL}$ [107]. As a result, in a structure that has a high level of membrane folding like the mitochondrial cristae, $\mathrm{CL}$ is fundamental for the maintenance of the membrane's morphological and functional integrities [108]. Namely, CL's involvements in the regulation of mitochondrial respiration, PKC activity, cytochrome c release, and much more, have been well researched and reviewed [106, 109, 
110]. Recently, it was reported that $C L$ also plays an essential role in regulating the stability and activity of the mammalian mitochondrial calcium uniporter (MCU). Specifically, a depletion of $\mathrm{CL}$ resulted in rapid turnover of MCU with reduced calcium transport across the mitochondrial membranes; this complication is implicated in the development of Barth syndrome [111]. Interestingly, although both $\mathrm{CL}$ and $\mathrm{PE}$ are conically-shaped and negative-curvature inducing in the PL bilayer, $\mathrm{CL}$ and PE depletion led to distinct pathophysiological outcomes in yeast cells despite lethality when both are depleted: a reduced mitochondrial $\mathrm{CL}$ disrupted the respiratory chain complexes, whereas a reduced mitochondrial PE stabilized the complexes while promoted formation of larger complexes [112]. This distinction between CL's and PE's roles in mitochondrial respiration regulation was further validated by the regulation of formation of the yeast mitochondrial adenosine $5^{\prime}$-diphosphate/adenosine 5 -triphosphate carrier (Aac) in the respiratory chain by $\mathrm{CL}$, since the depletion of such destabilized the respiratory chain protein complexes. The destabilization of the respiratory chain complexes directly disrupts $\Delta \Psi_{m}$ and mitochondrial bioenergetics, a demonstration of CL's critical role in maintaining mitochondrial functions [113]. On the other hand, $\mathrm{CL}$ is also a site for toxin insertion into the mitochondrial membrane, causing poreformation and membrane leak. Specifically, as related to PD, $\alpha$-synuclein ( $\alpha$-syn) inserted into the $\mathrm{IMM}$ in a CL-rich environment in vitro. It was observed that $\alpha$-syn could induce poration in the mitochondrial membrane and is detrimental to neuronal cells [114]. Therefore, maintenance of a low $\mathrm{CL}$ content on the OMM not only serves an important purpose in mitochondrial morphology, but exposure of $\mathrm{CL}$ on the outer OMM may increase the rate of PD development.

\subsection{Iron Accumulation and Oxidative Stress}

Iron accumulation in the brain and the resulted oxidative stress are often observed in neuropathology, together with autophagy and mitochondrial damage. Currently, there is no consensus on whether the iron accumulation is primary or secondary to other organellar damages. Nevertheless, both organellar damage and iron accumulation are hallmarks in the development of neurodegenerative diseases. A specific type of rare neurodegenerative disorders, coined neurodegeneration with brain iron accumulation (NBIA), are labelled by a common trait of iron deposit in the brain. Although more than ten genes had been identified as causative of NBIA, most of these genes are indeed unrelated to iron metabolism [115]. On the other hand, iron accumulation had also been observed in both chronic neurodegenerative diseases like AD, PD and ALS [116, 117], as well as acute neurodegenerations caused by traumatic brain injuries (TBI) [118], raising the speculation of brain iron deposits being a commonly observed outcome as a result of cellular dysfunctions. Whereas mitochondrial damage and autophagic dysregulation are usually observed with iron accumulation, oxidative stress is often present as a result of the excess free radical production by reduction of $\mathrm{H}_{2} \mathrm{O}_{2}$ by the iron ions [116, 119].

Among the NBIA, the currently identified deleterious mutations are in subtypes of related to iron homeostasis, coenzyme A synthesis, lipid metabolism, autophagy, and unknown functions as categorized by cellular pathways (reviewed in detail by Levi et al.). The Pla2g6 mutation and the C19orf12 mutation are involved in PL metabolism [120, 121]; specifically, a case with the absence of C19orf12 showed pathologies including Lewy bodies, tau proteins, tangles, and spheroids [122]. In addition to the current list of mutations causative of NBIA, we recently established the involvement of CTL1 in a novel early-onset neurodegenerative disease [7]. CTL1, a choline 
transporter expressed on both the plasma and mitochondrial membranes, is also pivotal for CNS health. Mutations in the SLC44A1 gene resulted in childhood-onset neurodegeneration accompanied with iron loading in the brain in two out of three separate mutations. Choline supplementation was able to restore membrane lipids and organelle functions and protect affected fibroblasts from iron loading, demonstrating choline deficiency as a cause for the disease. The important finding was that in the SLC44A1 mutant fibroblasts the major reduction was observed in the membrane PE not in PC. PC remained constant due to reduced PC degradation by PSS1 and increased formation by PE methylation [7]. Furthermore, we recently discovered that CTL1 also transport ethanolamine for PE synthesis, and that disruption in the PE homeostasis and consequently involving other PLs is indicative of an intricate internal system to maintain membrane PL balance [72].

\subsection{Significance of Phospholipid Dysregulation in Neurodegenerative Diseases}

Neurodegenerative diseases denote a series of events where the functions of the brain decline overtime due to the irreversible death of neuronal cells and the lack of regeneration thereof. Most often, a hallmark of such pathology is accompanied by the accumulation of toxic protein aggregates. As the central nervous is rich in lipids, the regulation of a functional lipid network is pivotal for the maintenance of neural health. In the following section, the implications of phospholipid dysregulation in each of the major neurodegenerative diseases are discussed in details.

\subsubsection{Alzheimer's Disease}

Alzheimer's disease (AD) has the clinical manifestation of mild cognitive impairment in behaviours with great heterogeneity; some of the areas are memory, task completion, comprehension, communication, mood and personality, and many more, with the most common manifestation being amnesia [123]. Although early diagnosis of AD dementia allows for timely preventative measures, usually the minor earliest symptoms remain undiagnosed for years until the disease progression may be irreversible worsened. Moreover, neuropathological development in the brains of $A D$ patients often precedes symptom onset, and neuropathological changes do not infer cognitive impairment in some cases [124]. The blurry line between clinical symptom onset and the biological definition of $A D$ hence is a constant subject of research and renewed knowledge. In 2018, the new National Institute on Aging-Alzheimer's Association research framework had been updated to introduce a more uniformed grading system and a common language for better communication among researchers. By this framework, $A D$ as a unique neurodegenerative disease is defined by the presence of extracellular $A \beta$ plaques, cytosolic tau tangles, and neurodegeneration, separate from other forms of dementia. Here, we will be focusing on the neuropathological manifestations of $A D$ as it relates to PL function in affected tissues [124].

While the presence of $A \beta$ and tau proteins have not been shown to be causal to AD development, both protein aggregates can cause neurotoxicity and neuronal cell death [124, 125]. The production of $A \beta$ is from the serial action of amyloidogenic cleavage of the amyloid precursor protein (APP) by first $\beta$-secretase then $\gamma$-secretase, resulting in the production of fibril-forming $A \beta$ peptides that are secreted into the extracellular space. Accumulation of the $A \beta$ peptides at a higher rate of clearance leads to the protein aggregate formation at the sites of hydrophobic residues, causing fibrillar plaques to form $[14,126]$. The dominant forms of the $A \beta$ peptides found in $A \beta$ fibrils are of $40(A \beta 40)$ 
and $42(A \beta 42)$ residues in size. The two peptides contribute to the formation of $A \beta$ fibrils differently, as the $A \beta 40$ peptide is more prominently expressed, but the $A \beta 42$ peptide is more likely to form fibrils [14]. While the expression of insoluble $A \beta$ fibrils is a hallmark in the brains of $A D$ patients, research had found that the soluble $A \beta$ oligomers as the precursor of $A \beta$ fibrils to be the more bioactive species in neurotoxicity $[127,128]$. Indeed, ample studies had demonstrated the neurotoxic effect of $A \beta$ oligomers: damages include nerve cell death, neurotic dystrophy in cultured neurons, synaptic dysfunction, disrupted insulin and calcium homeostasis in the brain, etc. [128130]. Clinically, these translate into symptoms such as memory loss, inability to form new memories, and a higher association of $A \beta$ oligomers, rather than $A \beta$ fibrils, with $A D$ development [129]. Moreover, both $A \beta$ oligomers and fibrils had been demonstrated to interact with tau proteins which are structurally associated with the microtubules under physiological condition [125]. Hyperphosphorylation of tau proteins occur with $A \beta$ overexpression, leading to dissociation and aggregation of tau proteins [131]. The pathological implications of these two neurotoxic protein aggregates include neuroinflammation, mitochondrial damage, elevated oxidative stress, apoptosis, iron accumulation, and much more [14, 117, 125, 132].

Numerous studies had demonstrated an altered PL profile in the CNS tissues of AD patients compared to physiologically healthy controls. Specifically, a significant difference was observed in the ratio of $\mathrm{PC} /$ lyso-PC between patients with either $\mathrm{MCl}$ or $\mathrm{AD}$, and healthy controls. This differentiation in PC/lyso-PC was proposed as an additional biomarker to the current ones for neuropathological diagnosis of $A D$, with an accuracy of $82 \%-85 \%$. This decreased ratio of PC/lysoPC was hypothesized as a result of increased phospholipase $A_{2}$ activities ( $\left.P L A_{2}\right)$, an enzyme catalyzing the cleavage of sn-2 fatty acid chain from PLs and is closely associated with AD pathology [68]. Additionally, other plasma PLs had also been proposed as biomarkers for the detection of preclinical $A D$, suggestive of disrupted $P L$ homeostasis in $A D$. In animal models experiencing repetitive traumatic brain injuries (TBI), elevated levels of $P E, P C$, and $\mathrm{PI}$ were observed in the hippocampus, and elevated levels of $\mathrm{PC}$ and $\mathrm{PI}$ were observed in the cortex, both at chronic time points post-injury [133]. On the contrary, there was a reduced level of $\mathrm{PC}, \mathrm{PI}$, and ether-PE in the cortex in the AD-phenotype model [134]. This result demonstrated a distinction between the PL profiles in the two neuropathologies, although repetitive TBI is a risk factor for AD development [134]. Interestingly, the increased PL levels in post-TBI models may suggest a higher likelihood of tau protein aggregation in neuronal tissues due to the increased phosphorylation by bioactive PLs. In a following animal trial with mice expressing all six human tau variants that experienced the same $\mathrm{TBI}$, acute and sub-acute increases in the PE, PC, and lyso-PE and lyso-PC levels were observed in the hippocampus and cortex. In comparison to the null Tau controls expressing a similar PL increase, the increase in PL levels in hTau mice may suggest that TBI, rather than tau pathology, is more causative of PL increase [133]. How tau pathology plays into the development of further neurodegeneration remains elusive. On the other hand, in a Chinese population, the PEMT G523A mutation was reported as a risk factor for $A D$, possibly due to lower $P C$ production and perhaps choline deficiency as a result of reduced PEMT activity [79]. As cholinergic signalling is a potential target of $A \beta$ oligomer toxicity, a compromised level of choline concentration may exacerbate dysregulation in cholinergic signalling that is central to proper brain functions [130].

Several PL-involved products were proposed as tentative treatment methods. For example, liposomes containing PC and PS were investigated as a candidate for anti-inflammatory treatments in the CNS, specifically targeting $A \beta$ induced-microglia $[135,136]$. Interestingly, anionic membrane 
surfaces such as supplied by PS had been previously shown to induce tau fibrilization and aggregation, promoting tau toxicity [137]. Therefore, the in vivo application of PC and PS containing liposomes in $A D$ would need further research.

\subsubsection{Parkinson's Disease}

Parkinson's disease (PD) is another prevalent neurodegenerative disease world-wide. The clinical manifestations of PD and other $\alpha$-synuclein ( $\alpha$-syn) related diseases such as dementia with Lewy bodies include impaired movement, tremor, micrographia, rigidity, as well as abnormal cognitive function [138]. PD is caused by neuronal cell death in the substantia nigra, as a result of the formation of insoluble protein aggregates of $\alpha$-syn. Insoluble $\alpha$-syn oligomers aggregate into inclusions of Lewy bodies (LB) in the cytoplasmic compartment, a hallmark of PD development [139]. The $\alpha$-syn aggregates impair neurotransmitter secretion, mitochondrial integrity and autophagy processes and they are able to self-propagate [139, 140].

Mitochondrial damage and autophagic dysfunction are the main mechanisms of pathological development in PD $[119,139,141]$. Mutations in PINK1 and Parkin had been prominently linked to autosomal recessive PD. As reviewed previously, mitochondria-related proteins implicated in PD include PINK1, Parkin, LRRK2, VDAC1, Tom40, HtrA2, and MCCC1. While some of them, such as PINK1, Parkin, Tom40 and HtrA2 are proteins directly functional in the mitochondria, proteins like LRRK2 have functions directly impact the maintenance of mitochondrial integrity $[95,119,142]$. Neuroepithelial stem cells with the LRRK2-G2019S mutation exhibit excess mitochondrial fragmentation, reduced $\triangle \Psi_{m}$ and respiration [143]. PINK1 and Parkin are involved in the MQC processes of fission and mitophagy, to eliminate mitochondria-induced cell death as described in Section IV. b).

Additional to the previously established processes, progressive changes in membrane lipids play a contributing role in PD pathologies. The amyloid cascade hypothesis (ACH) proposed that the progression of PD is due to the accumulation of spontaneous $\alpha$-syn aggregate formation [144]. In support of this hypothesis, neuronal $\alpha$-syn was reported to strongly interacts with phospholipids under nanomolar concentrations close to in physiological conditions, which leads to $\alpha$-syn neurotoxicity by increasing membrane permeability. The rate of $\alpha$-syn aggregation was positively associated with a protein-to-lipid ratio. As well, $\alpha$-syn aggregates were shown to spontaneously dissociate from the PL bilayer membranes, pointing to the critical role PL bilayer membranes play in the process of aggregate spreading and disease progression [144]. Moreover, the LB core is lipiddense, specifically of sphingomyelin and PC which may aid in further $\alpha$-syn aggregate formation [145]. In a Saccharomyces cerevisiae yeast PD model, the knockdown of PSD1 resulted in PE deficiency and consequently mitochondrial damage and ER stress, along with a three-fold increase in the $\alpha$-syn level [146]. The different changes in PLs, such as a decrease in the PC, PE and PI content, and an elevated PS level as well as the development of lipid droplets (LDs), as related to PD development are discussed in detail in a recent review [145]. Interestingly, a possible "bidirectional pathological interplay" between $\alpha$-syn and lipids was proposed where $\alpha$-syn aggregates have the propensity to increase lipid droplet formation and binding to them, the process reinforced by an increased MUFAs and PUFAs [145].

Genome-wide-association-studies (GWAS) had found genes from lipid metabolic pathways that are closely associated with disease onset and progression of PD. Lipid-related genes closely 
associated with PD development include genes encoding for glucocerebrosidase (GBA) and sterol regulatory element binding transcription factor 1 - retinoic acid induced 1 fusion protein SREBF1RAI1 [142]. As well, a recent study on 12 human cohorts identified SLC44A1 which encodes choline transporter-like protein 1 (CTL1) as a significant risk factor in later stages of PD [147]. The CTL1 plays a pivotal role in the process of choline transport for PC synthesis, as discussed in previous sections (sections III. b) and IV. c)). The three inherited homozygous frame-shift mutations in SLC44A1 gene (SLC44A1 AAsp517, SLC44A1 SSer126 and SLC44A1 DLys90) that cause a neurodegenerative disease with elements of childhood-onset parkinsonism and will be discussed in Section III. b).

\subsubsection{Huntington's Disease}

Huntington's disease (HD) is an autosomal dominant neurodegenerative disorder caused by a CAG expansion mutation in the gene encoding the huntingtin (HTT) protein. Although relatively rare, the clinical onset of the HD usually follows poor prognosis with death within the following one to two decades, accompanied by symptoms including motor and cognitive declines as well as disturbance in neuropsychiatry $[148,149]$. HD typically showed a large degree of variation in disease onset between individuals, an indication of environmental factors influencing the disease development. It is also generally believed that a longer CAG repeat is associated with earlier disease onset, and vice versa [15].

The pathological mechanism for HD development is generally attributed to aggregation of the cleaved-off $\mathrm{N}$-tails of mutant $\mathrm{HTT}$ ( $\mathrm{mHTT}$ ), which can affect a multitude of cellular processes [15]. Relatively well studies are the protein-protein interactions between $\mathrm{mHTT}$ and interaction partner proteins. These proteins are involved in cellular pathways including axonal transport, autophagy, palmitoylation, mitochondrial fusion and fission, etc. [150]. In particular, mTOR signaling is impaired in cell models expressing $\mathrm{mHTT}$, potentially by down regulating the striatal specific RAS-like protein Rhes [150]. mTOR signalling dysfunction has consequences for mitochondrial proliferation, lipid homeostasis, myelination, and neuronal cell proliferation [151, 152]. The mHTT aggregates can crossing over the nuclear membrane and directly interfere with gene transcription [150]. Moreover, it was recently discovered that not only can the $\mathrm{mHTT}$ protein, but also its mRNA, interfere with cellular processes via a gain-of-function mutation. Most proteins captured by the mHTT mRNA are spliceosomes, with the most notable target protein being PRPF8. This protein is a regulator of transcription factor CREB1 which has implications in HD development [153].

As new discoveries had been made on ferroptosis and iron accumulation, more and more associations had been drawn between HD pathology and mitochondrial dysfunction and oxidative damage. Indeed, iron accumulation and oxidative stress had been readily observed in both animal and human HD models. Although given the close relationship between a homeostatic PL pool and mitochondrial health, a lack of research can be found to draw a definitive connection between PL homeostasis and HD pathology. It was observed that supplementation with alpha-lipoic acid and acetyl-L-carnitine in rats could ameliorate 3-nitropropionic-acid induced HD pathology by restoring the mitochondrial lipid composition [154].

\subsubsection{Other Neurodegenerative Diseases}

Recently identified a novel childhood-onset neurodegenerative disease with Parkinsonian-like features caused by CTL1 deficiency, a result of loss-of-function, homozygous frameshift mutations 
in the SLC44A1 gene. Patients experienced delayed early development in motor and cognitive controls, and collectively experienced clinical symptoms entailing motor neuron dysfunctions including tremor, ataxia, dysarthria, difficulty swallowing, muscle weakness, strabismus, and decreased visual acuity [7]. CTL1 is choline and ethanolamine transporter (as discussed in previous sections) universally expressed in both the plasma and mitochondrial membranes for the transport of choline and ethanolamine for PC and PE synthesis by the Kennedy pathway [155]. Discovery of this connection between CTL1 deficiency and neurodegeneration had highlighted the significance of the membrane bilayer PL in the brain. This novel discovery is the first to draw a causal relationship between CTL1, PL, and neurodegenerative disease. It also demonstrates the interconversion between PLs and consolidates the significance of a balanced PL in maintaining the brain function.

$\mathrm{PE}$ dysregulation is also involved in the development of other neurological impairments. For example, a lack of PE-plasmalogens, was observed in Zellweger syndrome patients and most peroxisomal disorders lack plasmalogens $[156,157]$. As well, disruption of the CDP-ethanolamine pathway by partial Pcyt2 deficiency in humans led to the development of a complex hereditary spastic paraplegia, with disturbance shown in PL plasmalogens [9]. Furthermore, also recently found in humans was an autosomal recessive variant of the SELENO1 gene that encodes EPT, the enzyme catalyzing the last step of the CDP-ethanolamine pathway [8]. The clinical manifestations of this disease were strikingly similar to that with Pcyt2 deficiency, including mild intellectual disability, epilepsy, spasticity, and increased $\mathrm{T}_{2}$ intensity signal in the periventricular white matter. Interestingly, there was no detectable different PE levels in the patients' blood samples, indicating possible compensatory pathways for PE synthesis, potentially via the PSD pathway [8]. A similar compensatory effect was also observed in CTL1-deficient patients where the PL homeostasis instead of the PC content was disturbed [7]. Therefore, EPT deficiency, in combination with cases of Pcyt2deficiency in humans, point to the critical role of the CDP-ethanolamine pathway in lipid homeostasis.

PS is only produced from PC (PSS1) and PE (PSS2), and it converts to PE via PSD [34]. Loss-offunction splicing in transcription step of Pisd expression as a result of a homozygous mutation in intron 8 of PISD was causal to the development of Liberfarb syndrome, a multisystem disorder affecting the eyes, ears, bones, and brains of the patients [35]. More investigation is warranted to answer further questions regarding this rare genetic neuropathology including the cellular metabolic defects and potential treatments. Moreover, $[158,159]$ deficiency in both humans and mice developed polyneuropathy, hearing loss, ataxia, retinitis pigmentosa, and cataract (PHARC), an autosomal recessive disorder leading to senso-motor neurons demyelination, retinal dystrophy, and cerebellar atrophy. ABHD12 is a lysophosphatidylserine (LPS) lipase, hence the systematic knockdown likely caused disturbances in PS metabolism. Indeed, the knockdown of ABHD16A, a PS lipase, successfully decreased the elevation of the brain LPS level that is the outcome of ABHD12 knockout [160]. In combination with the deleterious effects from ABHD12 knockdown, a simultaneous LPCAT3 knockdown exacerbates the dysregulation by disruption of the conversion of LPS back to PS [159]. Together, the LPCAT3-ABHD12-ABHD16A axis demonstrated the involvement of PS in PHARC, an early onset multisystem disease. 


\subsubsection{Nutritional Treatments/ Supplementation}

As there is currently no cure for neurodegenerative diseases, treatments are aimed at preventing disease progression by reducing further neuronal damage and symptom management. Both physical and dietary interventions had been developed with various degrees of efficacy; for the purpose of this review, we will focus mainly on the scope of dietary interventions as a treatment for neurodegenerative diseases. Notably, as the gut-brain axis (GBA) continues to be shown as an important factor for brain health, the importance of a high dietary quality gains attention. The GBA is a pathway for the bidirectional communication between the gastrointestinal (GI) tract and the central nervous system, specifically into the cognitive and emotional centres of the brain. This crosstalk is conducted via several means including the nervous, immune, endocrine, and humoral pathways [161]. Specifically, the bottom-up communication from the gut to the brain are primarily done via the neuroimmune and neuroendocrine systems [162]. Substrates that act as transmitters in these networks comprise of short-chain-fatty-acids (SCFAs), tryptophan metabolites, and secondary bile acids [163]. Recently, this connection between the Gl tract and the brain had now been further extended to include influence from the gut microbiota, with dysbiosis as an important factor in many neurodegenerative diseases. In short, the increase in abundance of opportunistic gram-negative bacteria in the $\mathrm{Gl}$ tract is a common phenomenon across multiple neurodegenerative diseases such as AD and PD. This topic had been reviewed in detail by Gentile et al., with an effort to draw a clear connection between AD, PD, and ALS and the GBA and consequential dietary implications [163]. Therefore, dietary interventions that promote a healthy GI tract and gut microbiota is directly beneficial in prevention of neurodegenerative diseases.

Bioactive substances such as PUFAs and antioxidants also showed beneficial impacts when supplemented in both animal and human models with neurodegenerative diseases [163-165]. As dietary interventions are a sustainable way to yield significant results in the prevention of neurodegenerative disease progression, it is thus important to discuss the supplementation with nutraceutical substances as a treatment.

A popular treatment for neurodegenerative diseases are omega-3 PUFA supplementations, specifically of eicosapentaenoic acid (EPA) and docosahexaenoic acid (DHA) $[163,166]$. These are long chain fatty acids containing numerous unsaturated bonds, with the first double bond at the n3 position from the terminal methyl group, hence the name n-3 PUFAs [167]. These fatty acids are incorporate into the sn-2 position of PLs and increase membrane fluidity. Commonly believed to be present in high concentration in the brain, omega-3 PUFAs have recently been shown to be expressed at a moderate amount in the brain compared to in other tissues [63]. However, a decreased level of $n-3$ PUFAs in the brain is highly associated with numerous neurodegenerative diseases [168]. Supplementations with n-3 PUFAs had shown promising results as treatments against major NDs such as PD, AD, HD, ALS, in both human and animal models. Specifically, the supplementation of n-3 PUFAs had been suggested as a potential treatment for PD for its antioxidant, anti-inflammatory, cannabinoid-producing, and anti-apoptosis properties [169]. Indeed, n-3 PUFAs had demonstrated antioxidant effects in optic epithelial cells, through the activation of the nuclear factor erythroid 2-related factor (NRF2) antioxidant pathways [170]. Nrf2 is a master regulator for a series of antioxidant proteins such as the superoxide dismutase (SOD), the heme oxygenase (HO-1), the glutathione disulfide reductase (GSR), sulfiredoxin (Srx), thioredoxin (Trx), which participate in the antioxidant defense [171, 172]. Actions of Nrf2 against 
neurodegeneration had also been implicated by the reduced Nrf2 levels in ALS and PD-like mouse models; this is more detailed reviewed in [171]. Furthermore, post-mortem analysis of the brains of human ALS patients showed decreased levels of Nrf2 with elevated the Kelch-like ECH-associated protein 1 (KEAP1) [173]. Therefore, Nrf2 activation could be a potential pathway through which $n-$ 3 PUFAs could exert antioxidant effects. Additionally, EPA supplementation in human SH-SY 5Y and primary mesencephalic cells showed antioxidant activities via inhibition of NADPH oxidase and COX2 [174]; mTOR inhibition by n-3 PUFAs had also been proposed as a novel therapeutic approach for NDs [165]. As related to oxidative stress, neuroinflammation is also a hallmark of damage in the CNS. By reducing the oxidative stress in the nervous system, $n-3$ PUFAs can also provide antiinflammatory benefits, specifically via inhibition of pro-inflammatory genes such as COX-2, Nf-KB, CPLA2 [169]. Moreover, a prolonged n-3 PUFA supplementations could attenuate microglia activity and reduce TNF- $\alpha$ and IL-1 $\beta$ production which are pro-inflammatory cytokines $[169,175]$. As well, PPAR $\alpha$ was up-regulated by $n-3$ PUFA supplementation in patients with PD which could improve mitochondrial function [169]. Lastly, n-3 PUFA exhibits anti-apoptotic functions by increasing the Akt and Bcl-2 activity and subsequent mitophagy $[176,177]$. Caspase-3 activity and cytochrome c release had also shown reduction with DHA and EPA treatment, respectively, in human neuronal cell models [169].

As lipid metabolic regulation is highly correlated with the health of the CNS, and as an altered phospholipid profile had been observed in the brains of ND patients, maintaining physiological PL homeostasis as a therapeutic approach had been a subject of research interest. Notably, choline supplementation as a mean to promote PC and acetylcholine syntheses had shown beneficial effects against NDs. Indeed, the neuroprotective actions of choline had been reviewed extensively in the past, especially pointing to their effects in membrane integrity and cholinergic neuron firing, as well as their roles as a methyl donor and epigenetic regulator of gene expressions [178, 179]. A life-long choline supplementation in an $A D$ mouse model had been shown to ameliorate disease progression by reducing the amyloidogenic splicing of APP and thus decrease the A $\beta$ load, reduce neuroinflammation by microglial activity, and downregulate the $\alpha 7 \mathrm{nAch}$ and $\alpha 1$ receptors [180]. As well, beneficial outcome was shown in choline treatment in primary mutant fibroblasts from CTL1deficient patients experiencing childhood-onset neurodegeneration [7].

In addition to choline, different PLs had also been investigated as treatments for neurodegeneration. PL containing ether bonds particularly in PE (PE-plasmalogens) are deficient in PD patients and can be rescued by oral supplementation of PE plasmalogens [181]. PS combined with ferulic acid and curcumin significantly improved cognitive function in an AD-like mouse model with a reduced $A \beta$ load, reduced neuroinflammation and an increased acetylcholine level [182]. Lastly, PL lipid vesicle for drug-delivery across the blood-brain barrier (BBB) are also a form of lipid molecule for increased n-3 PUFA brain availability [183]. PS is used to construct nanoliposomes enclosing metformin, a drug commonly used to treat type II diabetes but also showed inhibitory effects against $A \beta$ and tau protein productions [136]. Moreover, artificial unilamellar vesicles composed of anionic PLs had been shown to reduce $A \beta$ fibrillation in vitro, evidencing the significance of lipid composition in $A \beta$ fibril formation and interactions with the plasma membrane [184]. In terms of $n-3$ PUFA dietary enrichment, PL-containing krill oil can provide neuroprotective effects due to its high n-3 PUFA content delivered in the form of PLs by attaching to the sn-2 positions [185]. 
As discussed in previous chapters, PL homeostasis is critical for the maintenance of proper brain functions through processes including autophagy, mitochondrial functions, and oxidative stress. Therefore, therapeutic approaches to strengthen the regulation of PL homeostasis is yet another angle to combat neurodegeneration. Current understanding of PL supplementation as a treatment for NDs is largely limited to the structural role of PL in cell membranes or as a vessel for drug transportation. However, it is within the functions of PL in maintaining cell membrane integrity can we draw a connection between the protective role of $\mathrm{PL}$ supplementation in both metabolic syndromes and NDs. Namely, mitochondrial dysfunction is a common occurrence for all NDs, and it is one of the main pathways through which PL supplementation can ameliorate disease progression. For instance, hypermetabolism is present in $50 \%$ of ALS patients as a result of mitochondrial dysfunction due to oxidative damage in motor neurons and skeletal muscles; although optimizing carbohydrate utilization is the more direct therapeutic target for ALS, administration of PLS is complimentary in rescuing mitochondrial integrity [186]. Furthermore, lipid supplementations with PLs and plasmalogen had both demonstrated beneficial effects in ameliorating $A D$ and PD symptoms [157, 181]. Dietary supplementation with food-derived PLs in Lipid Replacement Therapy (LRT) had been implicated as a way to treat a large array of chronic diseases including NDs, specifically targeting mitochondrial dysfunction especially when administrated with a cocktail of Lcarnitine, $\alpha$-lipoic acid (ALA), coenzyme-Q10, reduced nicotinamide adenine dinucleotide (NADH) $[187,188]$. Indeed, it was demonstrated that the metabolic fate of orally consumed diacyl-PLs is largely through the enzymatic breakdown of diacyl-PLs to monoacyl-PLs, leading to the uptake by enterocytes via passive diffusion [189]. Although, there exists other transport systems such as pinocytosis or the direct insertion of individual PLS into the outer lipid bilayer [188]. The incorporation of orally ingested PLs into tissues had been shown in rats to be near $100 \%$, deeming oral administration an efficient way of PL supplementation [188]. On the other hand, mitochondrial $\mathrm{CL}$ had been shown as a binding site for $\alpha$-syn in the development of PD, warranting it a target for therapeutic approaches [190]. The use of drugs such as elamipretide which can selectively bind to $\mathrm{CL}$ had been demonstrated for its effects in preventing the formation of the complex cyt c-CL [191].

Several vitamins, especially lipid soluble vitamin $E$, had also undergone investigation for their neuroprotective effects. Vitamin $E$ is a strong antioxidant with positive neuroprotective activities. Vitamin E could restore corticostriatal synaptic plasticity via rescue of mitochondrial functions in PINK1 knockout mice as a PD model [192]. As well, in aged DNA-repair deficient mice, administration of a high level of vitamin E reduced p53-positive apoptotic cells [164]. The synergic effect of vitamin $E$ and PUFAs is evident, as vitamin E can protect PL-bound PUFAs from oxidative damage, which in turn helps maintain membrane integrity [193]. In animal models with TBI-related dementia, vitamin E supplementation showed some beneficial effects although more in-depth studies are needed for conclusive results [193]. In particular, quantification of an adequate dosage of vitamin $E$ as a treatment for TBI-related dementia needs further research. Additionally, new evidence had suggested a new view on the neuroprotective effects of vitamin $E$, broadening its bioactivity from simply antioxidant to modulating of lipid metabolism by gene expression regulation. Specifically, vitamin $\mathrm{E}$ seems to have a brain-specific metabolism in that, it undergoes less autooxidation in the brain [194]. The natural metabolite of vitamin E, $\alpha$-tocopheryl $(\alpha-T)$, had been demonstrated to influence the lipid free radical flux by formation of the more stable radical, $\alpha-\mathrm{TO}^{\circ}$, and the downstream regeneration of $\alpha-\mathrm{TOH}$ [195]. Moreover, another endogenous metabolite of vitamin E, the alpha-tocopheryl hydroquinone $(\alpha-T Q)$, was recently shown to be an even more potent 
ferroptosis inhibitor via the inhibition of 15-lipoxygenase, which could have neuroprotective benefits by preventing neuronal cell death [196]. Lastly, it had been demonstrated that the $\alpha-T$ metabolite of vitamin E could bind to lipoxygenases and phospholipase A2 (PLA2) in a competitive manner against PUFAs and PLs, respectively, that can inhibit the peroxidation and oxidation of the lipids [197]. In the context of PL lipidomics, the regulation of PLA2 activity by vitamin E can be a modulator of the PL composition in the brain which was implicated in many NDs [198]. Altogether, the molecular activities of vitamin $E$ and its derivatives in the brain in reducing oxidative stress renders it a major participant in combating neuroinflammation [194, 199]. Translating the molecular activities of vitamin $E$ into in vivo animal studies, a recent study demonstrated another vitamin $E$ metabolite, garcinoic acid (GA), was effective in preventing $\beta$-amyloid deposition in the mouse brain [200]. Therefore, it is also worth noting that, vitamin E metabolites instead of the parent form, also exhibit neuroprotective activities.

\subsubsection{Conclusion}

Previously thought as a family of strictly protein-induced diseases, the development of NDs in fact involves a complex series of dysfunction in multiple cellular pathways. PLs are the fundamental building blocks for the plasma and organelle membranes, and their participation in cellular pathways is imperative for the cell's survival. Due to the intricate network of PL synthesis and interconversion, disruption of a homeostatic level of tissue-specific PL species ratios yields detrimental outcomes. As strong associations are drawn between brain and even whole-body PL levels and ND development, the negatively causative effects of PL dysregulation are being revealed. Cellular processes such as mitochondrial respiration, autophagy, oxidative stress management all require PLs to proceed, and the dysfunction of them can result in neuronal cell death. With the rise of newer omics technologies, genes and proteins in PL metabolism that are associated with NDs are being discovered at a rate faster than ever before. It is thus important to identify more PL metabolic genes in relation to NDs and thus warrants further studies.

\section{Author Contributions}

$X . R$, Quan performed the literature review, formulated the outline, and prepared the first draft and the final draft and incorporated changes in multiple drafts. M. Bakovic helped in the manuscript conceptualization, made outline suggestions, edited all drafts, reviewed the final manuscript, and responded to comments of the reviewers.

\section{Competing Interests}

The authors have declared that no competing interests exist.

\section{References}

1. Van Meer G, Voelker DR, Feigenson GW. Membrane lipids: Where they are and how they behave. Nat Rev Mol Cell Biol. 2008; 9: 112-124.

2. Drin G. Topological regulation of lipid balance in cells. Annu Rev Biochem. 2014; 83: 51-77.

3. Calzada E, Onguka O, Claypool SM. Chapter two - phosphatidylethanolamine metabolism in health and disease. Int Rev Cell Mol Biol. 2016; 321: 29-88. 
4. Fullerton MD, Hakimuddin F, Bakovic M. Developmental and metabolic effects of disruption of the mouse CTP: Phosphoethanolamine cytidylyltransferase gene (Pcyt2). Mol Cell Biol. 2007; 27: 3327-3336.

5. Steenbergen R, Nanowski TS, Beigneux A, Kulinski A, Young SG, Vance JE. Disruption of the phosphatidylserine decarboxylase gene in mice causes embryonic lethality and mitochondrial defects. J Biol Chem. 2005; 280: 40032-40040.

6. Miyazaki C, Saitoh M, Itoh M, Yamashita S, Miyagishi M, Takashima S, et al. Altered phospholipid molecular species and glycolipid composition in brain, liver and fibroblasts of Zellweger syndrome. Neurosci Lett. 2013; 552: 71-75.

7. Fagerberg CR, Taylor A, Distelmaier F, Schrøder HD, Kibæk M, Wieczorek D, et al. Choline transporter-like 1 deficiency causes a new type of childhood-onset neurodegeneration. Brain. 2020; 143: 94-111.

8. Ahmed MY, Al-Khayat A, Al-Murshedi F, Al-Futaisi A, Chioza BA, Fernandez-Murray JP, et al. A mutation of EPT1 (SELENOI) underlies a new disorder of Kennedy pathway phospholipid biosynthesis. Brain. 2017; 140: 547-554.

9. Vaz FM, McDermott JH, Alders M, Wortmann SB, Kölker S, Pras-Raves ML, et al. Mutations in PCYT2 disrupt etherlipid biosynthesis and cause a complex hereditary spastic paraplegia. Brain. 2019; 142: 3382-3397.

10. Rickman OJ, Baple EL, Crosby AH. Lipid metabolic pathways converge in motor neuron degenerative diseases. Brain. 2020; 143: 1073-1087.

11. Cunnane SC, Trushina E, Morland C, Prigione A, Casadesus G, Andrews ZB, et al. Brain energy rescue: An emerging therapeutic concept for neurodegenerative disorders of ageing. Nat Rev Drug Discov. 2020; 19: 609-633.

12. Mattson M, Magnus T. Ageing and neuronal vulnerability. Nat Rev Neurosci. 2006; 7: 278-294.

13. Lau A, So RW, Lau HH, Sang JC, Ruiz-Riquelme A, Fleck SC, et al. $\alpha$-Synuclein strains target distinct brain regions and cell types. Nat Neurosci. 2020; 23: 21-31.

14. Tiwari S, Atluri V, Kaushik A, Yndart A, Nair M. Alzheimer's disease: Pathogenesis, diagnostics, and therapeutics. Int J Nanomedicine. 2019; 14: 5541-5554.

15. Saudou F, Humbert S. The biology of huntingtin. Neuron. 2016; 89: 910-926.

16. Gillette-Guyonnet S, Secher M, Vellas B. Nutrition and neurodegeneration: Epidemiological evidence and challenges for future research. Br J Clin Pharmacol. 2013; 75: 738-755.

17. Serrano-Pozo A, Frosch MP, Masliah E, Hyman BT. Neuropathological alterations in Alzheimer disease. Cold Spring Harb Perspect Med. 2011; 1: a006189.

18. Gibellini F, Smith TK. The Kennedy pathway-de novo synthesis of phosphatidylethanolamine and phosphatidylcholine. IUBMB Life. 2010; 62: 414-428.

19. Ingólfsson HI, Melo MN, Van Eerden FJ, Arnarez C, Lopez CA, Wassenaar TA, et al. Lipid organization of the plasma membrane. J Am Chem Soc. 2014; 136: 14554-14559.

20. Vance DE, Vance JE. Chapter 8 - phospholipid biosynthesis in eukaryotes. In: Biochemistry of Lipids, lipoproteins and membranes. 5th ed. Netherlands: Elsevier; 2008.

21. Leventis PA, Grinstein S. The distribution and function of phosphatidylserine in cellular membranes. Annu Rev Biophys. 2010; 39: 407-427.

22. Mejia EM, Nguyen H, Hatch GM. Mammalian cardiolipin biosynthesis. Chem Phys Lipids. 2014; 179: 11-16.

23. Moessinger C, Klizaite K, Steinhagen A, Philippou-Massier J, Shevchenko A, Hoch M, et al. Two 
different pathways of phosphatidylcholine synthesis, the Kennedy Pathway and the Lands Cycle, differentially regulate cellular triacylglycerol storage. BMC Cell Biol. 2014; 15: 43.

24. Fagone $\mathrm{P}$, Jackowski S. Phosphatidylcholine and the CDP-choline cycle. Biochim Biophys Acta Mol Cell Biol Lipids. 2013; 1831: 523-532.

25. Schenkel LC, Bakovic M. Formation and regulation of mitochondrial membranes. Int J Cell Biol. 2014; 2014: 709828.

26. van der Veen JN, Kennelly JP, Wan S, Vance JE, Vance DE, Jacobs RL. The critical role of phosphatidylcholine and phosphatidylethanolamine metabolism in health and disease. Biochim Biophys Acta Biomembr. 2017; 1859: 1558-1572.

27. Michel V, Singh RK, Bakovic M. The impact of choline availability on muscle lipid metabolism. Food Funct. 2011; 2: 53-62.

28. Cornell RB, Ridgway ND. CTP: Phosphocholine cytidylyltransferase: Function, regulation, and structure of an amphitropic enzyme required for membrane biogenesis. Prog Lipid Res. 2015; 59: 147-171.

29. Strakova J, Demizieux L, Campenot RB, Vance DE, Vance JE. Involvement of CTP: Phosphocholine cytidylyltransferase- $\beta 2$ in axonal phosphatidylcholine synthesis and branching of neurons. Biochim Biophys Acta Mol Cell Biol Lipids. 2011; 1811: 617-625.

30. Jackowski S, Rehg JE, Zhang YM, Wang J, Miller K, Jackson P, et al. Disruption of CCT $\beta 2$ expression leads to gonadal dysfunction. Mol Cell Biol. 2004; 24: 4720-4733.

31. Marcucci H, Paoletti L, Jackowski S, Banchio C. Phosphatidylcholine biosynthesis during neuronal differentiation and its role in cell fate determination. J Biol Chem. 2010; 285: 2538225393.

32. Reo NV, Adinehzadeh M, Foy BD. Kinetic analyses of liver phosphatidylcholine and phosphatidylethanolamine biosynthesis using 13C NMR spectroscopy. Biochim Biophys Acta Mol Cell Biol Lipids. 2002; 1580: 171-188.

33. Vance DE. Phospholipid methylation in mammals: From biochemistry to physiological function. Biochim Biophys Acta Biomembr. 2014; 1838: 1477-1487.

34. Vance JE, Tasseva $G$. Formation and function of phosphatidylserine and phosphatidylethanolamine in mammalian cells. Biochim Biophys Acta Mol Cell Biol Lipids. 2013; 1831: 543-554.

35. Peter VG, Quinodoz M, Pinto-Basto J, Sousa SB, Di Gioia SA, Soares G, et al. The Liberfarb syndrome, a multisystem disorder affecting eye, ear, bone, and brain development, is caused by a founder pathogenic variant in the PISD gene. Genet Med. 2019; 21: 2734-2743.

36. Shindou H, Hishikawa D, Harayama T, Yuki K, Shimizu T. Recent progress on acyl CoA: Lysophospholipid acyltransferase research. J Lipid Res. 2009; 50: S46-S51.

37. Basu P, Alibhai FJ, Tsimakouridze EV, Singh RK, Paglialunga S, Holloway GP, et al. Male-specific cardiac dysfunction in CTP: Phosphoethanolamine cytidylyltransferase (Pcyt2)-deficient mice. Mol Cell Biol. 2015; 35: 2641-2657.

38. Fullerton MD, Hakimuddin F, Bonen A, Bakovic M. The development of a metabolic disease phenotype in CTP: Phosphoethanolamine cytidylyltransferase-deficient mice. J Biol Chem. 2009; 284: 25704-25713.

39. Leonardi R, Frank MW, Jackson PD, Rock CO, Jackowski S. Elimination of the CDP-ethanolamine pathway disrupts hepatic lipid homeostasis. J Biol Chem. 2009; 284: 27077-27089.

40. Selathurai A, Kowalski GM, Burch ML, Sepulveda P, Risis S, Lee-Young RS, et al. The CDP- 
ethanolamine pathway regulates skeletal muscle diacylglycerol content and mitochondrial biogenesis without altering insulin sensitivity. Cell Metab. 2015; 21: 718-730.

41. Poloumienko A, Coté A, Ten Quee AT, Zhu L, Bakovic M. Genomic organization and differential splicing of the mouse and human Pcyt2 genes. Gene. 2004; 325: 145-155.

42. Pavlovic Z, Singh RK, Bakovic M. A novel murine CTP: Phosphoethanolamine cytidylyltransferase splice variant is a post-translational repressor and an indicator that both cytidylyltransferase domains are required for activity. Gene. 2014; 543: 58-68.

43. Tie A, Bakovic M. Alternative splicing of CTP: Phosphoethanolamine cytidylyltransferase produces two isoforms that differ in catalytic properties. J Lipid Res. 2007; 48: 2172-2181.

44. Balla T, Sengupta N, Kim YJ. Lipid synthesis and transport are coupled to regulate membrane lipid dynamics in the endoplasmic reticulum. Biochim Biophys Acta Mol Cell Biol Lipids. 2020; 1865: 158461.

45. Kainu V, Hermansson M, Hänninen S, Hokynar K, Somerharju P. Import of phosphatidylserine to and export of phosphatidylethanolamine molecular species from mitochondria. Biochim Biophys Acta Mol Cell Biol Lipids. 2013; 1831: 429-437.

46. Kuge O, Saito K, Kojima M, Akamatsu Y, Nishijima M. Post-translational processing of the phosphatidylserine decarboxylase gene product in Chinese hamster ovary cells. Biochem J. 1996; 319: 33-38.

47. Friedman JR, Kannan M, Toulmay A, Jan CH, Weissman JS, Prinz WA, et al. Lipid homeostasis is maintained by dual targeting of the mitochondrial PE biosynthesis enzyme to the ER. Dev Cell. 2018; 44: 261-270.

48. Arikketh D, Nelson R, Vance JE. Defining the importance of phosphatidylserine synthase-1 (PSS1): Unexpected viability of PSS1-deficient mice. J Biol Chem. 2008; 283: 12888-12897.

49. Kuge O, Saito K, Nishijima M. Cloning of a Chinese hamster ovary ( $\mathrm{CHO}$ ) cDNA encoding phosphatidylserine synthase (PSS) II, overexpression of which suppresses the phosphatidylserine biosynthetic defect of a PSS I-lacking mutant of CHO-K1 cells. J Biol Chem. 1997; 272: 19133-19139.

50. Stone SJ, Vance JE. Phosphatidylserine synthase-1 and-2 are localized to mitochondriaassociated membranes. J Biol Chem. 2000; 275: 34534-34540.

51. Vance JE. Historical perspective: Phosphatidylserine and phosphatidylethanolamine from the 1800s to the present. J Lipid Res. 2018; 59: 923-944.

52. Stone SJ, Cui Z, Vance JE. Cloning and expression of mouse liver phosphatidylserine synthase-1 cDNA: Overexpression in rat hepatoma cells inhibits the CDP-ethanolamine pathway for phosphatidylethanolamine biosynthesis. J Biol Chem. 1998; 273: 7293-7302.

53. Kimura AK, Kim HY. Phosphatidylserine synthase 2: High efficiency for synthesizing phosphatidylserine containing docosahexaenoic acid. J Lipid Res. 2013; 54: 214-222.

54. Kuge O, Hasegawa K, Saito K, Nishijima M. Control of phosphatidylserine biosynthesis through phosphatidylserine-mediated inhibition of phosphatidylserine synthase $\mathrm{I}$ in Chinese hamster ovary cells. Proc Natl Acad Sci U S A. 1998; 95: 4199-4203.

55. Kuge O, Saito K, Nishijima M. Control of phosphatidylserine synthase II activity in Chinese hamster ovary cells. J Biol Chem. 1999; 274: 23844-23849.

56. Sousa SB, Jenkins D, Chanudet E, Tasseva G, Ishida M, Anderson G, et al. Gain-of-function mutations in the phosphatidylserine synthase 1 (PTDSS1) gene cause Lenz-Majewski syndrome. Nat Genet. 2014; 46: 70-76. 
57. Tanguy $E$, Wang $Q$, Moine $H$, Vitale N. Phosphatidic acid: From pleiotropic functions to neuronal pathology. Front Cell Neurosci. 2019; 13: 2.

58. Gimeno RE, Cao J. Thematic review series: Glycerolipids. Mammalian glycerol-3-phosphate acyltransferases: New genes for an old activity. J Lipid Res. 2008; 49: 2079-2088.

59. Blunsom NJ, Cockcroft S. CDP-diacylglycerol synthases (CDS): Gateway to phosphatidylinositol and cardiolipin synthesis. Front Cell Dev Biol. 2020; 8: 63.

60. Ye C, Shen Z, Greenberg ML. Cardiolipin remodeling: A regulatory hub for modulating cardiolipin metabolism and function. J Bioenerg Biomembr. 2016; 48: 113-123.

61. Blunsom NJ, Cockcroft S. Phosphatidylinositol synthesis at the endoplasmic reticulum. Biochim Biophys Acta Mol Cell Biol Lipids. 2020; 1865: 158471.

62. Hernandez-Diaz S, Soukup SF. The role of lipids in autophagy and its implication in neurodegeneration. Cell Stress. 2020; 4: 167-186.

63. Kuschner CE, Yagi T, Kim J. Chapter 2 - thinking in polyunsaturated fatty acids, phospholipids, and the brain. In: Omega Fatty Acids in Brain and Neurological Health. 2nd ed. Cambridge: Academic Press; 2019. pp.21-32.

64. Maccarrone M, Dainese E, Oddi S. Intracellular trafficking of anandamide: New concepts for signaling. Trends Biochem Sci. 2010; 35: 601-608.

65. Ingólfsson HI, Carpenter TS, Bhatia H, Bremer PT, Marrink SJ, Lightstone FC. Computational lipidomics of the neuronal plasma membrane. Biophys J. 2017; 113: 2271-2280.

66. Sabogal-Guáqueta AM, Villamil-Ortiz JG, Arias-Londoño JD, Cardona-Gómez GP. Inverse phosphatidylcholine/phosphatidylinositol levels as peripheral biomarkers and phosphatidylcholine/lysophosphatidylethanolamine-phosphatidylserine as hippocampal indicator of postischemic cognitive impairment in rats. Front Neurosci. 2018; 12: 989.

67. Pollard AK, Ortori CA, Stöger R, Barrett DA, Chakrabarti L. Mouse mitochondrial lipid composition is defined by age in brain and muscle. Aging. 2017; 9: 986-995.

68. Klavins K, Koal T, Dallmann G, Marksteiner J, Kemmler G, Humpel C. The ratio of phosphatidylcholines to lysophosphatidylcholines in plasma differentiates healthy controls from patients with Alzheimer's disease and mild cognitive impairment. Alzheimers Dement. 2015; 1: 295-302.

69. Murphy EJ, Schapiro MB, Rapoport SI, Shetty HU. Phospholipid composition and levels are altered in down syndrome brain. Brain Res. 2000; 867: 9-18.

70. Michel V, Yuan Z, Ramsubir S, Bakovic M. Choline transport for phospholipid synthesis. Exp Biol Med. 2006; 231: 490-504.

71. Hou Y, Pinheiro J, Sahm F, Reuss DE, Schrimpf D, Stichel D, et al. Papillary glioneuronal tumor (PGNT) exhibits a characteristic methylation profile and fusions involving PRKCA. Acta Neuropathol. 2019; 137: 837-846.

72. Taylor A, Grapentine S, Ichhpuniani J, Bakovic M. The novel roles of choline transporter-like 1 and 2 in ethanolamine transport. BioRxiv. 2020. Doi: 10.1101/2020.08.27.270223.

73. Li L, Ding Z, Pang TL, Zhang B, Li CH, Liang AM, et al. Defective minor spliceosomes induce SMAassociated phenotypes through sensitive intron-containing neural genes in Drosophila. Nat Commun. 2020; 11: 5608.

74. Ando H, Horibata Y, Yamashita S, Oyama T, Sugimoto H. Low-density lipoprotein and oxysterols suppress the transcription of CTP: Phosphoethanolamine cytidylyltransferase in vitro. Biochim Biophys Acta Mol Cell Biol Lipids. 2010; 1801: 487-495. 
75. Pavlovic Z, Bakovic M. Regulation of phosphatidylethanolamine homeostasis-the critical role of CTP: Phosphoethanolamine cytidylyltransferase (Pcyt2). Int J Mol Sci. 2013; 14: 2529-2550.

76. Ando H, Horibata Y, Aoyama C, Shimizu H, Shinohara Y, Yamashita S, et al. Side-chain oxysterols suppress the transcription of CTP: Phosphoethanolamine cytidylyltransferase and 3-hydroxy-3methylglutaryl-CoA reductase by inhibiting the interaction of p300 and NF-Y, and H3K27 acetylation. J Steroid Biochem Mol Biol. 2019; 195: 105482.

77. Griffiths WJ, Wang Y. Oxysterol research: A brief review. Biochem Soc Trans. 2019; 47: 517-526.

78. Steenbergen R, Nanowski TS, Nelson R, Young SG, Vance JE. Phospholipid homeostasis in phosphatidylserine synthase-2-deficient mice. Biochim Biophys Acta Mol Cell Biol Lipids. 2006; 1761: 313-323.

79. Bi XH, Zhao HL, Zhang ZX, Zhang JW. PEMT G523A (V175M) is associated with sporadic Alzheimer's disease in a Chinese population. J Mol Neurosci. 2012; 46: 505-508.

80. Peker N, Gozuacik D. Autophagy as a cellular stress response mechanism in the nervous system. J Mol Biol. 2020; 432: 2560-2588.

81. Cuervo AM, Wong E. Chaperone-mediated autophagy: Roles in disease and aging. Cell Res. 2014; 24: 92-104.

82. Li WW, Li J, Bao JK. Microautophagy: Lesser-known self-eating. Cell Mol Life Sci. 2012; 69: 11251136.

83. Yu L, Chen Y, Tooze SA. Autophagy pathway: Cellular and molecular mechanisms. Autophagy. 2018; 14: 207-215.

84. Hara T, Nakamura K, Matsui M, Yamamoto A, Nakahara Y, Suzuki-Migishima R, et al. Suppression of basal autophagy in neural cells causes neurodegenerative disease in mice. Nature. 2006; 441: 885-889.

85. Dikic I, Elazar Z. Mechanism and medical implications of mammalian autophagy. Nat Rev Mol Cell Biol. 2018; 19: 349-364.

86. Schütter M, Giavalisco P, Brodesser S, Graef M. Local fatty acid channeling into phospholipid synthesis drives phagophore expansion during autophagy. Cell. 2020; 180: 135-149.e14.

87. Girardi JP, Pereira L, Bakovic M. De novo synthesis of phospholipids is coupled with autophagosome formation. Med Hypotheses. 2011; 77: 1083-1087.

88. Andrejeva G, Gowan S, Lin G, Wong Te Fong AC, Shamsaei E, Parkes HG, et al. De novo phosphatidylcholine synthesis is required for autophagosome membrane formation and maintenance during autophagy. Autophagy. 2020; 16: 1044-1060.

89. Kim DI, Lee KH, Gabr AA, Choi GE, Kim JS, Ko SH, et al. A $\beta$-Induced Drp1 phosphorylation through Akt activation promotes excessive mitochondrial fission leading to neuronal apoptosis. Biochim Biophys Acta Mol Cell Res. 2016; 1863: 2820-2834.

90. Young JE, Martinez RA, La Spada AR. Nutrient deprivation induces neuronal autophagy and implicates reduced insulin signaling in neuroprotective autophagy activation. J Biol Chem. 2009; 284: 2363-2373.

91. van der Heide LP, Ramakers GM, Smidt MP. Insulin signaling in the central nervous system: Learning to survive. Prog Neurobiol. 2006; 79: 205-221.

92. Menon D, Salloum D, Bernfeld E, Gorodetsky E, Akselrod A, Frias MA, et al. Lipid sensing by mTOR complexes via de novo synthesis of phosphatidic acid. J Biol Chem. 2017; 292: 6303-6311.

93. Rugarli El, Langer T. Mitochondrial quality control: A matter of life and death for neurons. EMBO J. 2012; 31: 1336-1349. 
94. Sinha S, Manoj N. Molecular evolution of proteins mediating mitochondrial fission-fusion dynamics. FEBS Lett. 2019; 593: 703-718.

95. Ge P, Dawson VL, Dawson TM. PINK1 and Parkin mitochondrial quality control: A source of regional vulnerability in Parkinson's disease. Mol Neurodegener. 2020; 15: 20.

96. Di Pietro V, Lazzarino G, Amorini AM, Signoretti S, Hill LJ, Porto E, et al. Fusion or fission: The destiny of mitochondria in traumatic brain injury of different severities. Sci Rep. 2017; 7: 9189.

97. Seabright AP, Fine NH, Barlow JP, Lord SO, Musa I, Gray A, et al. AMPK activation induces mitophagy and promotes mitochondrial fission while activating TBK1 in a PINK1-Parkin independent manner. FASEB J. 2020; 34: 6284-6301.

98. Lee $\mathrm{Y}$, Stevens DA, Kang SU, Jiang H, Lee YI, Ko HS, et al. PINK1 primes Parkin-mediated ubiquitination of PARIS in dopaminergic neuronal survival. Cell Rep. 2017; 18: 918-932.

99. Zheng L, Bernard-Marissal N, Moullan N, D'Amico D, Auwerx J, Moore DJ, et al. Parkin functionally interacts with PGC-1 $\alpha$ to preserve mitochondria and protect dopaminergic neurons. Hum Mol Genet. 2017; 26: 582-598.

100.Peng K, Xiao J, Yang L, Ye F, Cao J, Sai Y. Mutual antagonism of PINK1/Parkin and PGC-1 $\alpha$ contributes to maintenance of mitochondrial homeostasis in rotenone-induced neurotoxicity. Neurotox Res. 2019; 35: 331-343.

101.Bader V, Winklhofer KF. Mitochondria at the interface between neurodegeneration and neuroinflammation. Semin Cell Dev Biol. 2020; 99: 163-171.

102. Burman JL, Pickles S, Wang C, Sekine S, Vargas JN, Zhang Z, et al. Mitochondrial fission facilitates the selective mitophagy of protein aggregates. J Cell Biol. 2017; 216: 3231-3247.

103.Selathurai A, Kowalski GM, Mason SA, Callahan DL, Foletta VC, Della Gatta PA, et al. Phosphatidylserine decarboxylase is critical for the maintenance of skeletal muscle mitochondrial integrity and muscle mass. Mol Metab. 2019; 27: 33-46.

104.Gohil VM, Zhu L, Baker CD, Cracan V, Yaseen A, Jain M, et al. Meclizine inhibits mitochondrial respiration through direct targeting of cytosolic phosphoethanolamine metabolism. J Biol Chem. 2013; 288: 35387-35395.

105. Fontana D, Mauri M, Renso R, Docci M, Crespiatico I, Røst LM, et al. ETNK1 mutations induce a mutator phenotype that can be reverted with phosphoethanolamine. Nat Commun. 2020; 11: 5938.

106.Pennington ER, Funai K, Brown DA, Shaikh SR. The role of cardiolipin concentration and acyl chain composition on mitochondrial inner membrane molecular organization and function. Biochim Biophys Acta Mol Cell Biol Lipids. 2019; 1864: 1039-1052.

107. Beltrán-Heredia E, Tsai FC, Salinas-Almaguer S, Cao FJ, Bassereau P, Monroy F. Membrane curvature induces cardiolipin sorting. Commun Biol. 2019; 2: 225.

108. Ikon N, Ryan RO. Cardiolipin and mitochondrial cristae organization. Biochim Biophys Acta Biomembr. 2017; 1859: 1156-1163.

109. Dudek J. Role of cardiolipin in mitochondrial signaling pathways. Front Cell Dev Biol. 2017; 5: 90.

110. Maguire JJ, Tyurina YY, Mohammadyani D, Kapralov AA, Anthonymuthu TS, Qu F, et al. Known unknowns of cardiolipin signaling: The best is yet to come. Biochim Biophys Acta Mol Cell Biol Lipids. 2017; 1862: 8-24.

111.Ghosh S, Ball WB, Madaris TR, Srikantan S, Madesh M, Mootha VK, et al. An essential role for cardiolipin in the stability and function of the mitochondrial calcium uniporter. Proc Natl Acad Sci U S A. 2020; 117: 16383-16390. 
112. Böttinger L, Horvath SE, Kleinschroth T, Hunte C, Daum G, Pfanner N, et al. Phosphatidylethanolamine and cardiolipin differentially affect the stability of mitochondrial respiratory chain supercomplexes. J Mol Biol. 2012; 423: 677-686.

113.Senoo N, Kandasamy S, Ogunbona OB, Baile MG, Lu Y, Claypool SM. Cardiolipin, conformation, and respiratory complex-dependent oligomerization of the major mitochondrial ADP/ATP carrier in yeast. Sci Adv. 2020; 6: eabb0780.

114.Ghio S, Camilleri A, Caruana M, Ruf VC, Schmidt F, Leonov A, et al. Cardiolipin promotes poreforming activity of alpha-synuclein oligomers in mitochondrial membranes. ACS Chem Neurosci. 2019; 10: 3815-3829.

115. Levi S, Tiranti V. Neurodegeneration with brain iron accumulation disorders: Valuable models aimed at understanding the pathogenesis of iron deposition. Pharmaceuticals. 2019; 12: 27.

116. Guan H, Yang H, Yang M, Yanagisawa D, Bellier JP, Mori $M$, et al. Mitochondrial ferritin protects $\mathrm{SH}-\mathrm{SY} 5 \mathrm{Y}$ cells against $\mathrm{H}_{2} \mathrm{O}_{2}$-induced oxidative stress and modulates $\alpha$-synuclein expression. Exp Neurol. 2017; 291: 51-61.

117. Hadzhieva M, Kirches E, Mawrin C. Iron metabolism and the role of iron in neurodegenerative disorders. Neuropathol Appl Neurobiol. 2014; 40: 240-257.

118.Zhang L, Wang H, Zhou X, Mao L, Ding K, Hu Z. Role of mitochondrial calcium uniporter-mediated $\mathrm{Ca}^{2+}$ and iron accumulation in traumatic brain injury. J Cell Mol Med. 2019; 23: 2995-3009.

119.Gómez-Sánchez R, Bravo-San Pedro JM, Gegg ME, González-Polo RA, Fuentes JM. Mitochondria: Key organelle in Parkinson's disease. Parkinsons Dis. 2016; 2016: 6230370.

120.Cheon Y, Kim HW, Igarashi M, Modi HR, Chang L, Ma K, et al. Disturbed brain phospholipid and docosahexaenoic acid metabolism in calcium-independent phospholipase A2-VIA (iPLA2 $\beta$ )knockout mice. Biochim Biophys Acta Mol Cell Biol Lipids. 2012; 1821: 1278-1286.

121. Deus CM, Yambire KF, Oliveira PJ, Raimundo N. Mitochondria-lysosome crosstalk: From physiology to neurodegeneration. Trends Mol Med. 2020; 26: 71-88.

122. Hartig $M B$, luso $A$, Haack $T$, Kmiec $T$, Jurkiewicz $E$, Heim $K$, et al. Absence of an orphan mitochondrial protein, C19orf12, causes a distinct clinical subtype of neurodegeneration with brain iron accumulation. Am J Hum Genet. 2011; 89: 543-550.

123. Atri A. The Alzheimer's disease clinical spectrum: Diagnosis and management. Med Clin North Am. 2019; 103: 263-293.

124.Jack Jr CR, Bennett DA, Blennow K, Carrillo MC, Dunn B, Haeberlein SB, et al. NIA-AA research framework: Toward a biological definition of Alzheimer's disease. Alzheimers Dement. 2018; 14: 535-562.

125. Bloom GS. Amyloid- $\beta$ and tau: The trigger and bullet in Alzheimer disease pathogenesis. JAMA Neurol. 2014; 71: 505-508.

126. Crews L, Masliah E. Molecular mechanisms of neurodegeneration in Alzheimer's disease. Hum Mol Genet. 2010; 19: R12-R20.

127.Jin M, Shepardson N, Yang T, Chen G, Walsh D, Selkoe DJ. Soluble amyloid $\beta$-protein dimers isolated from Alzheimer cortex directly induce Tau hyperphosphorylation and neuritic degeneration. Proc Natl Acad Sci U S A. 2011; 108: 5819-5824.

128. Yang T, Li S, Xu H, Walsh DM, Selkoe DJ. Large soluble oligomers of amyloid $\beta$-protein from Alzheimer brain are far less neuroactive than the smaller oligomers to which they dissociate. J Neurosci. 2017; 37: 152-163.

129.Arbel-Ornath M, Hudry E, Boivin JR, Hashimoto T, Takeda S, Kuchibhotla KV, et al. Soluble 
oligomeric amyloid- $\beta$ induces calcium dyshomeostasis that precedes synapse loss in the living mouse brain. Mol Neurodegener. 2017; 12: 27.

130.Mroczko B, Groblewska M, Litman-Zawadzka A, Kornhuber J, Lewczuk P. Amyloid $\beta$ oligomers (AßOs) in Alzheimer's disease. J Neural Transm. 2018; 125: 177-191.

131. Lloret A, Fuchsberger T, Giraldo E, Vina J. Molecular mechanisms linking amyloid $\beta$ toxicity and Tau hyperphosphorylation in Alzheimer' s disease. Free Radic Biol Med. 2015; 83: 186-191.

132. Nikseresht S, Bush Al, Ayton S. Treating Alzheimer's disease by targeting iron. Br J Pharmacol. 2019; 176: 3622-3635.

133. Ojo JO, Algamal M, Leary $P$, Abdullah L, Mouzon B, Evans JE, et al. Disruption in brain phospholipid content in a humanized tau transgenic model following repetitive mild traumatic brain injury. Front Neurosci. 2018; 12: 893.

134.Ojo JO, Algamal M, Leary P, Abdullah L, Mouzon B, Evans JE, et al. Converging and differential brain phospholipid dysregulation in the pathogenesis of repetitive mild traumatic brain injury and Alzheimer's disease. Front Neurosci. 2019; 13: 103.

135. Hashioka S, Han YH, Fujii S, Kato T, Monji A, Utsumi H, et al. Phosphatidylserine and phosphatidylcholine-containing liposomes inhibit amyloid $\beta$ and interferon- $\gamma$-induced microglial activation. Free Radic Biol Med. 2007; 42: 945-954.

136.Saffari PM, Alijanpour S, Takzaree N, Sahebgharani M, Etemad-Moghadam S, Noorbakhsh F, et al. Metformin loaded phosphatidylserine nanoliposomes improve memory deficit and reduce neuroinflammation in streptozotocin-induced Alzheimer's disease model. Life Sci. 2020; 255: 117861.

137. Chirita CN, Necula M, Kuret J. Anionic micelles and vesicles induce tau fibrillization in vitro. J Biol Chem. 2003; 278: 25644-25650.

138. Rao SS, Hofmann LA, Shakil A. Parkinson's disease: Diagnosis and treatment. Am Fam Physician. 2006; 74: 2046-2054.

139. Sironi L, Restelli LM, Tolnay M, Neutzner A, Frank S. Dysregulated interorganellar crosstalk of mitochondria in the pathogenesis of Parkinson's disease. Cells. 2020; 9: 233.

140.Lashuel HA, Overk CR, Oueslati A, Masliah E. The many faces of $\alpha$-synuclein: From structure and toxicity to therapeutic target. Nat Rev Neurosci. 2013; 14: 38-48.

141.Corti O, Blomgren K, Poletti A, Beart PM. Autophagy in neurodegeneration: New insights underpinning therapy for neurological diseases. J Neurochem. 2020; 154: 354-371.

142. Nalls MA, Pankratz N, Lill CM, Do CB, Hernandez DG, Saad M, et al. Large-scale meta-analysis of genome-wide association data identifies six new risk loci for Parkinson's disease. Nat Genet. 2014; 46: 989-993.

143. Walter J, Bolognin S, Antony PM, Nickels SL, Poovathingal SK, Salamanca L, et al. Neural stem cells of Parkinson's disease patients exhibit aberrant mitochondrial morphology and functionality. Stem Cell Rep. 2019; 12: 878-889.

144.Lv Z, Hashemi M, Banerjee S, Zagorski K, Rochet JC, Lyubchenko YL. Assembly of $\alpha$-synuclein aggregates on phospholipid bilayers. Biochim Biophys Acta Proteins Proteom. 2019; 1867: 802812.

145. Fanning S, Selkoe D, Dettmer U. Parkinson's disease: Proteinopathy or lipidopathy? NPJ Parkinsons Dis. 2020; 6: 3.

146.Wang S, Zhang S, Liou LC, Ren Q, Zhang Z, Caldwell GA, et al. Phosphatidylethanolamine deficiency disrupts $\alpha$-synuclein homeostasis in yeast and worm models of Parkinson disease. 
Proc Natl Acad Sci U S A. 2014; 111: E3976-E3985.

147.Iwaki H, Blauwendraat C, Leonard HL, Kim JJ, Liu G, Maple-Grødem J, et al. Genome-wide association study of Parkinson's disease progression biomarkers in 12 longitudinal patients' cohorts. BioRxiv. 2019. Doi: 10.1101/585836.

148. Mi Y, Gao X, Xu H, Cui Y, Zhang Y, Gou X. The emerging roles of ferroptosis in Huntington's disease. Neuromolecular Med. 2019; 21: 110-119.

149.Scahill RI, Zeun P, Osborne-Crowley K, Johnson EB, Gregory S, Parker C, et al. Biological and clinical characteristics of gene carriers far from predicted onset in the Huntington's disease young adult study (HD-YAS): A cross-sectional analysis. Lancet Neurol. 2020; 19: 502-512.

150. Wanker EE, Ast A, Schindler F, Trepte P, Schnoegl S. The pathobiology of perturbed mutant huntingtin protein-protein interactions in Huntington's disease. J Neurochem. 2019; 151: 507519.

151.Figlia G, Gerber D, Suter U. Myelination and mTOR. Glia. 2018; 66: 693-707.

152.Saxton RA, Sabatini DM. mTOR signaling in growth, metabolism, and disease. Cell. 2017; 168: 960-976.

153.Schilling J, Broemer M, Atanassov I, Duernberger Y, Vorberg I, Dieterich C, et al. Deregulated splicing is a major mechanism of RNA-induced toxicity in Huntington's disease. J Mol Biol. 2019; 431: 1869-1877.

154. Mehrotra A, Sood A, Sandhir R. Mitochondrial modulators improve lipid composition and attenuate memory deficits in experimental model of Huntington's disease. Mol Cell Biochem. 2015; 410: 281-292.

155. Michel V, Bakovic M. The solute carrier 44A1 is a mitochondrial protein and mediates choline transport. FASEB J. 2009; 23: 2749-2758.

156.Jo DS, Cho DH. Peroxisomal dysfunction in neurodegenerative diseases. Arch Pharm Res. 2019; 42: 393-406.

157.Paul S, Lancaster GI, Meikle PJ. Plasmalogens: A potential therapeutic target for neurodegenerative and cardiometabolic disease. Prog Lipid Res. 2019; 74: 186-195.

158.Blankman JL, Long JZ, Trauger SA, Siuzdak G, Cravatt BF. ABHD12 controls brain lysophosphatidylserine pathways that are deregulated in a murine model of the neurodegenerative disease PHARC. Proc Natl Acad Sci U S A. 2013; 110: 1500-1505.

159.Ichu TA, Reed A, Ogasawara D, Ulanovskaya O, Roberts A, Aguirre CA, et al. ABHD12 and LPCAT3 interplay regulates a lyso-phosphatidylserine-C20: 4 phosphatidylserine lipid network implicated in neurological disease. Biochemistry. 2020; 59: 1793-1799.

160. Kamat SS, Camara K, Parsons WH, Chen DH, Dix MM, Bird TD, et al. Immunomodulatory lysophosphatidylserines are regulated by ABHD16A and ABHD12 interplay. Nat Chem Biol. 2015; 11: 164-171.

161.Carabotti M, Scirocco A, Maselli MA, Severi C. The gut-brain axis: Interactions between enteric microbiota, central and enteric nervous systems. Ann Gastroenterol. 2015; 28: 203-209.

162. Martin CR, Osadchiy V, Kalani A, Mayer EA. The brain-gut-microbiome axis. Cell Mol Gastroenterol Hepatol. 2018; 6: 133-148.

163.Gentile F, Doneddu PE, Riva N, Nobile-Orazio E, Quattrini A. Diet, microbiota and brain health: Unraveling the network intersecting metabolism and neurodegeneration. Int J Mol Sci. 2020; 21: 7471.

164.La Fata G, van Vliet N, Barnhoorn S, Brandt RM, Etheve S, Chenal E, et al. Vitamin E 
supplementation reduces cellular loss in the brain of a premature aging mouse model. J Prev Alzheimers Dis. 2017; 4: 226-235.

165. Shirooie S, Nabavi SF, Dehpour AR, Belwal T, Habtemariam S, Argüelles S, et al. Targeting mTORs by omega-3 fatty acids: A possible novel therapeutic strategy for neurodegeneration? Pharmacol Res. 2018; 135: 37-48.

166. Ajibawo-Aganbi U, Saleem S, Khan SZ, Veliginti S, Bastidas MV, Lungba RM, et al. Can nutritional adequacy help evade neurodegeneration in older age? A review. Cureus. 2020; 12: e10921.

167. Shahidi F, Ambigaipalan P. Omega-3 polyunsaturated fatty acids and their health benefits. Annu Rev Food Sci Technol. 2018; 9: 345-381.

168. Avallone $R$, Vitale $G$, Bertolotti M. Omega-3 fatty acids and neurodegenerative diseases: New evidence in clinical trials. Int J Mol Sci. 2019; 20: 4256.

169. Li P, Song C. Potential treatment of Parkinson's disease with omega-3 polyunsaturated fatty acids. Nutr Neurosci. 2020; 23: 1-12.

170. Tourtas T, Birke MT, Kruse FE, Welge-Lüssen UC, Birke K. Preventive effects of omega-3 and omega- 6 Fatty acids on peroxide mediated oxidative stress responses in primary human trabecular meshwork cells. PLoS ONE. 2012; 7: e31340.

171. Ma Q. Role of nrf2 in oxidative stress and toxicity. Annu Rev Pharmacol Toxicol. 2013; 53: 401426.

172.Stefanson A, Bakovic M. Dietary polyacetylene falcarinol upregulated intestinal heme oxygenase-1 and modified plasma cytokine profile in late phase lipopolysaccharide-induced acute inflammation in CB57BL/6 mice. Nutr Res. 2020; 80: 89-105.

173.Sarlette A, Krampfl K, Grothe C, Neuhoff NV, Dengler R, Petri S. Nuclear erythroid 2-related factor 2-antioxidative response element signaling pathway in motor cortex and spinal cord in amyotrophic lateral sclerosis. J Neuropathol Exp Neurol. 2008; 67: 1055-1062.

174.Luchtman DW, Meng Q, Wang X, Shao D, Song C. Omega-3 fatty acid eicospentaenoic acid attenuates MPP+-induced neurodegeneration in fully differentiated human SH-SY $5 Y$ and primary mesencephalic cells. J Neurochem. 2013; 124: 855-868.

175.Ji A, Diao H, Wang X, Yang R, Zhang J, Luo W, et al. N-3 polyunsaturated fatty acids inhibit lipopolysaccharide-induced microglial activation and dopaminergic injury in rats. Neurotoxicology. 2012; 33: 780-788.

176. Chen X, Pan Z, Fang Z, Lin W, Wu S, Yang F, et al. Omega-3 polyunsaturated fatty acid attenuates traumatic brain injury-induced neuronal apoptosis by inducing autophagy through the upregulation of SIRT1-mediated deacetylation of Beclin-1. J Neuroinflammation. 2018; 15: 310.

177. Kitagishi Y, Nakanishi A, Ogura Y, Matsuda S. Dietary regulation of PI3K/AKT/GSK-3 $\beta$ pathway in Alzheimer's disease. Alzheimers Res Ther. 2014; 6: 35.

178. Bekdash RA. Choline, the brain and neurodegeneration: Insights from epigenetics. Front Biosci. 2018; 23: 1113-1143.

179. Blusztajn JK, Slack BE, Mellott TJ. Neuroprotective actions of dietary choline. Nutrients. 2017; 9 : 815.

180. Velazquez R, Ferreira E, Knowles S, Fux C, Rodin A, Winslow W, et al. Lifelong choline supplementation ameliorates Alzheimer's disease pathology and associated cognitive deficits by attenuating microglia activation. Aging Cell. 2019; 18: e13037.

181. Mawatari S, Ohara S, Taniwaki Y, Tsuboi Y, Maruyama T, Fujino T. Improvement of blood plasmalogens and clinical symptoms in Parkinson's disease by oral administration of ether 
phospholipids: A preliminary report. Parkinsons Dis. 2020; 2020: 2671070.

182. Okuda M, Fujita $Y$, Sugimoto $H$. The additive effects of low dose intake of ferulic acid, phosphatidylserine and curcumin, not alone, improve cognitive function in APPswe/PS1dE9 transgenic mice. Biol Pharm Bull. 2019; 42: 1694-1706.

183. Yalagala PR, Sugasini D, Dasarathi S, Pahan K, Subbaiah PV. Dietary lysophosphatidylcholine-EPA enriches both EPA and DHA in the brain: Potential treatment for depression [S]. J Lipid Res. 2019; 60: 566-578.

184. Chaparro Sosa AF, de Oliveira da Silva SM, Morgan GP, Schwartz DK, Kaar JL. Mixed phospholipid vesicles catalytically inhibit and reverse amyloid fibril formation. J Phys Chem Lett. 2020; 11: 7417-7422.

185. Tomé-Carneiro J, Crespo MC, Burgos-Ramos E, Tomas-Zapico C, García-Serrano A, Castro-Gómez $P$, et al. Buttermilk and krill oil phospholipids improve hippocampal insulin resistance and synaptic signaling in aged rats. Mol Neurobiol. 2018; 55: 7285-7296.

186. Noland D, Drisko JA, Wagner L. Integrative and functional medical nutrition therapy: Principles and practices. Switzerland: Springer Nature; 2020.

187. Nicolson GL, Settineri R, Ellithorpe RR. Neurodegenerative and fatiguing illnesses, infections and mitochondrial dysfunction: Use of natural supplements to improve mitochondrial function. Funct Food Health Dis. 2014; 4: 23-65.

188. Nicolson GL, Ash ME. Lipid replacement therapy: A natural medicine approach to replacing damaged lipids in cellular membranes and organelles and restoring function. Biochim Biophys Acta Biomembr. 2014; 1838: 1657-1679.

189.van Hoogevest P. Review-an update on the use of oral phospholipid excipients. Eur J Pharm Sci. 2017; 108: 1-12.

190.Gilmozzi V, Gentile G, Castelo-Rueda MP, Hicks AA, Pramstaller P, Zanon A, et al. Interaction of alpha-synuclein with lipids: Mitochondrial cardiolipin as a critical player in the pathogenesis of Parkinson's disease. Front Neurosci. 2020; 14: 578993.

191. Paradies G, Paradies V, Ruggiero FM, Petrosillo G. Role of cardiolipin in mitochondrial function and dynamics in health and disease: Molecular and pharmacological aspects. Cells. 2019; 8: 728.

192.Schirinzi T, Martella G, Imbriani P, Di Lazzaro G, Franco D, Colona VL, et al. Dietary Vitamin E as a protective factor for Parkinson's disease: Clinical and experimental evidence. Front Neurol. 2019; 10: 148.

193.Dobrovolny J, Smrcka M, Bienertova-Vasku J. Therapeutic potential of vitamin E and its derivatives in traumatic brain injury-associated dementia. Neurol Sci. 2018; 39: 989-998.

194.Ambrogini P, Torquato P, Bartolini D, Albertini MC, Lattanzi D, Di Palma M, et al. Excitotoxicity, neuroinflammation and oxidant stress as molecular bases of epileptogenesis and epilepsyderived neurodegeneration: The role of vitamin E. Biochim Biophys Acta Mol Basis Dis. 2019; 1865: 1098-1112.

195.Shah R, Shchepinov MS, Pratt DA. Resolving the role of lipoxygenases in the initiation and execution of ferroptosis. ACS Cent Sci. 2018; 4: 387-396.

196. Hinman A, Holst CR, Latham JC, Bruegger JJ, Ulas G, McCusker KP, et al. Vitamin E hydroquinone is an endogenous regulator of ferroptosis via redox control of 15-lipoxygenase. PLoS ONE. 2018; 13: e0201369.

197. Chandra V, Jasti J, Kaur P, Betzel C, Srinivasan A, Singh TP. First structural evidence of a specific inhibition of phospholipase $A 2$ by $\alpha$-tocopherol (vitamin E) and its implications in inflammation: 
Crystal structure of the complex formed between phospholipase A2 and $\alpha$-tocopherol at $1.8 \AA$ resolution. J Mol Biol. 2002; 320: 215-222.

198. Khanna S, Parinandi NL, Kotha SR, Roy S, Rink C, Bibus D, et al. Nanomolar vitamin E $\alpha-$ tocotrienol inhibits glutamate-induced activation of phospholipase $A 2$ and causes neuroprotection. J Neurochem. 2010; 112: 1249-1260.

199. Hermann PM, Watson SN, Wildering WC. Phospholipase A2-nexus of aging, oxidative stress, neuronal excitability, and functional decline of the aging nervous system? Insights from a snail model system of neuronal aging and age-associated memory impairment. Front Genet. 2014; 5: 419.

200. Marinelli R, Torquato P, Bartolini D, Mas-Bargues C, Bellezza G, Gioiello A, et al. Garcinoic acid prevents $\beta$-amyloid $(A \beta)$ deposition in the mouse brain. J Biol Chem. 2020; 295: 11866-11876.

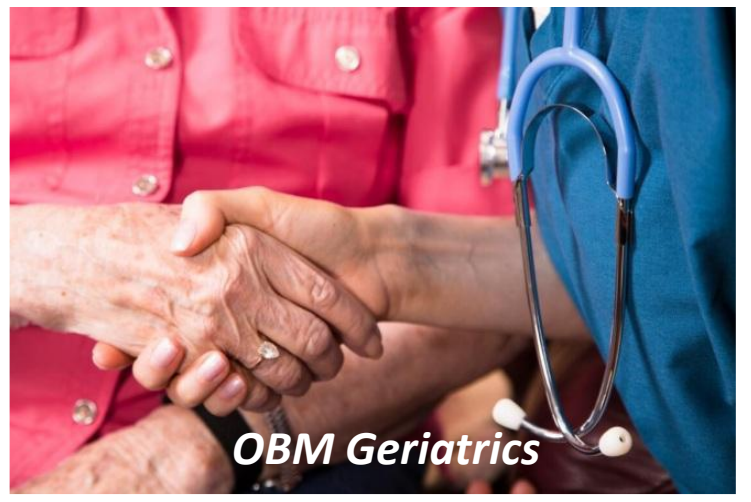

Enjoy OBM Geriatrics by:

1. Submitting a manuscript

2. Joining in volunteer reviewer bank

3. Joining Editorial Board

4. Guest editing a special issue

For more details, please visit:

http://www.lidsen.com/journals/geriatrics 